\title{
Quench dynamics near a quantum critical point: application to the sine-Gordon model
}

\author{
C. De Grandi ${ }^{1}$, V. Gritsev ${ }^{2}$, and A. Polkovnikov ${ }^{1}$ \\ ${ }^{1}$ Department of Physics, Boston University, 590 Commonwealth Avenue, Boston, MA 02215, USA \\ ${ }^{2}$ Department of Physics, University of Fribourg, Chemin du Musée 3, 1700 Fribourg, Switzerland
}

\begin{abstract}
We discuss the quench dynamics near a quantum critical point focusing on the sine-Gordon model as a primary example. We suggest a unified approach to sudden and slow quenches, where the tuning parameter $\lambda(t)$ changes in time as $\lambda(t) \sim v t^{r}$, based on the adiabatic expansion of the excitation probability in powers of $v$. We show that the universal scaling of the excitation probability can be understood through the singularity of the generalized adiabatic susceptibility $\chi_{2 r+2}(\lambda)$, which for sudden quenches $(r=0)$ reduces to the fidelity susceptibility. In turn this class of susceptibilities is expressed through the moments of the connected correlation function of the quench operator. We analyze the excitations created after a sudden quench of the cosine potential using a combined approach of form-factors expansion and conformal perturbation theory for the low-energy and highenergy sector respectively. We find the general scaling laws for the probability of exciting the system, the density of excited quasiparticles, the entropy and the heat generated after the quench. In the two limits where the sine-Gordon model maps to hard core bosons and free massive fermions we provide the exact solutions for the quench dynamics and discuss the finite temperature generalizations.
\end{abstract}

\section{INTRODUCTION}

The Sine-Gordon (SG) model is one of the few examples of exactly solvable models both in the classical and in the quantum case. In the classical limit, an arbitrary initial perturbation splits into solitons and breathers, which are infinitely long lived excitations (see e.g. Ref. [1]). In the quantum regime these solitons and breathers are quantized and form a discrete spectrum (for each momentum state) [2, 3]. This model found numerous applications in various fields of physics including statistical physics, condensed-matter physics, atomic physics and high-energy physics. Its integrability allows to solve various equilibrium and non-equilibrium situations and gain important insights into phenomena ranging from classical and quantum phase transitions [4 $[-6$ ] to quench dynamics of coupled superfluids [7, 8].

For the purposes of this work we will keep in mind the applications of this model to one-dimensional quantum systems. Thus a natural low-energy theory describing a one-dimensional interacting gas of bosons or fermions is the Luttinger liquid model, which is nothing but a scalar free $1+1$ dimensional bosonic theory [5]. The Luttinger liquid is often unstable to various perturbations opening a gap in the system coming either from the external potential (periodic [9] or disordered [10]) or the tunneling coupling between multiple Luttinger liquids [11]. In these situations the most relevant perturbations appear in the form of the lowest harmonic of the cosine potential allowed by the symmetry [6, 12] leading to the sine-Gordon Hamiltonian:

$$
H=\frac{1}{2} \int d x\left[\left(\partial_{x} \phi(x)\right)^{2}+(\Pi(x))^{2}-4 \lambda \cos (\beta \phi(x))\right] .
$$

In cold-atom systems this Hamiltonian can be implemented through optical lattices [9, 13], it also emerges naturally in the context of interacting quantum wires [5], 14], mesoscopic superconducting junctions [15], spin chains [5, 6] and many others.

The recent experimental developments in cold-atoms [16, 17] prompted a rapidly growing theoretical interest to study the dynamics of quantum systems. A particular attention was paid to such issues as sudden quench dynamics [18 24, thermalization in integrable and nonintegrable systems 25 28] and slow dynamics in isolated systems near quantum critical points 29 31]. The latter works illustrated the universal scaling of the density of quasiparticles generated due to nonadiabatic transitions during the crossing of quantum phase transitions. The universal properties of quantum critical points in equilibrium extend to the dynamics and allow one to make universal predictions on the defects and energy generation similar to the Kibble-Zurek relations 32, 33] for slow dynamics near quantum critical points [34 44].

In the preceding work [45] we showed that a similar universality also persists for a sudden quench dynamics near quantum critical points if the quench amplitude is sufficiently small. There we analyzed the general scaling behavior of different quantities following a sudden quench of the amplitude $\lambda_{f}$ starting from the critical point $\lambda_{i}=0$. We presented some general arguments for the scaling of various quantities such as the probability of exciting the system $P_{\mathrm{ex}}$, the density of excited quasiparticles $n_{\mathrm{ex}}$, the (diagonal) entropy density $S_{d}[46]$, and the heat density $Q$ [47], with the quench amplitude and the system size. We showed that the corresponding scaling laws are universal and can be described by two critical exponents characterizing the quantum critical point: the dynamical exponent $z$ and the correlation length exponent $\nu$. We also argued that the scaling behavior for sudden quenches is smoothly connected to the scaling behavior of similar quantities for slow quenches, where the tuning parameter is turned on as an arbitrary power of time [48, 49]: $\lambda(t) \sim v t^{r}$, where $v$ is a small parameter. In particular, in the limit $r \rightarrow 0$ this parameter becomes the quench amplitude: $v=\lambda_{f}$.

The goal of this paper is to verify how the general ar- 
guments of Ref. [45] work in practice. We will use the sine-Gordon model as a primary example. Performing explicit calculations based on the adiabatic perturbation theory, form-factor approach, and conformal perturbation theory we will illustrate how these scalings are realized for the case of quenching the cosine potential and highlight various subtleties which can emerge due to possible ultraviolet or infrared divergencies. In the two limits where the SG model reduces to free massive bosons and fermions we will extend the analysis to higher dimensions and again verify the general predictions of Ref. [45]. In these two limits we will be able to generalize the results to finite temperatures and see how the quasiparticle statistics affects the scaling of various quantities.

The paper is organized as follows: in Sec. II we review the general scaling results presented in Ref. [45] introducing a generalized framework that allows us to describe sudden quenches and slow quenches on the same footing. In Sec. III we describe the basic properties of the SG model. Then in Secs. IV and $[\mathrm{V}$ we analyze the quench dynamics in the SG model using the adiabatic perturbation theory. In Sec. VI we present the exact analysis of quenches in the two noninteracting limits of the SG model corresponding to the free massive bosons and free massive fermions. In Sec. VID we furthermore extend this analysis to finite temperature quenches. Finally in Sec. VII we discuss some interesting relations between quench dynamics, fidelity susceptibility and geometric tensors.

\section{GENERALIZED FRAMEWORK FOR THE ANALYSIS OF QUENCH DYNAMICS: HIERARCHY OF SUSCEPTIBILITIES}

In this section we will illustrate a generalized approach to study the scaling laws of the quantities of interest after performing a quench. As in Ref. [45] we consider a $d$ dimensional system described by a Hamiltonian $H(\lambda)=$
$H_{0}+\lambda V$, where $H_{0}$ is the Hamiltonian corresponding to a quantum critical point (QCP) and $V$ is a relevant (or marginal) perturbation. In particular, we consider the processes where the coupling changes near the critical point as a power law in time:

$$
\lambda(t)=v \frac{t^{r}}{r !} \Theta(t)
$$

where $v$ is a small parameter that we define as $v \equiv$ $\left.\frac{d^{r} \lambda}{d t^{r}}\right|_{t=0}$ and $\Theta$ is the step function, so that the coupling starts changing with time as $t^{r}$ near the critical point. On the same footing we can consider the opposite processes, where quenches stop at the critical point as:

$$
\lambda(t)=v \frac{\left(t_{f}-t\right)^{r}}{r !} \Theta\left(t_{f}-t\right) .
$$

The analysis also extends to cyclic processes where the dynamical evolution starts and ends at the quantum critical point. E.g. $\lambda(t) \approx v t^{r}\left(t_{f}-t\right)^{r} /\left(r ! t_{f}^{r}\right)$. In all these cases $v$ plays the role of the adiabatic parameter such that $v \rightarrow 0$ corresponds to the adiabatic limit. In particular, if the system is initially prepared in the ground state then in the limit $v \rightarrow 0$ it will remain in the ground state. The physical meaning of $v$ is quite transparent. For sudden quenches $r=0$, it corresponds to the quench amplitude $v=\lambda_{f}$; for linear quenches $r=1$, it describes the quench rate $v=\dot{\lambda}$; for quadratic quenches $r=2$, it describes the acceleration near the quantum critical point: $v=\ddot{\lambda}$ and so. The smallness of $v$ suggests the possibility of using it as an expansion parameter. Relying on the adiabatic perturbation theory one can indeed write an expansion in the powers of $v$. The details of this approach can be found in Refs. [50, [51]. We only quote the final expression for the transition amplitude to the excited state $|n\rangle$ as a result of this process (see e.g. Eq. (19) in Ref. [50] or Eq. (18) in Ref. [51]):

$$
\begin{aligned}
& \left.\alpha_{n}\left(t_{f}\right) \approx\left[i \frac{\left\langle n\left|\partial_{t}\right| 0\right\rangle}{E_{n}(t)-E_{0}(t)}-\frac{1}{E_{n}(t)-E_{0}(t)} \frac{d}{d t} \frac{\left\langle n\left|\partial_{t}\right| 0\right\rangle}{E_{n}(t)-E_{0}(t)}+\ldots\right] \mathrm{e}^{i\left(\Theta_{n}(t)-\Theta_{0}(t)\right)}\right|_{t_{i}} ^{t_{f}} \\
& =\left.\left[i \dot{\lambda} \frac{\left\langle n\left|\partial_{\lambda}\right| 0\right\rangle}{E_{n}(\lambda)-E_{0}(\lambda)}-\ddot{\lambda} \frac{\left\langle n\left|\partial_{\lambda}\right| 0\right\rangle}{\left(E_{n}(\lambda)-E_{0}(\lambda)\right)^{2}}-\dot{\lambda}^{2} \frac{1}{E_{n}(\lambda)-E_{0}(\lambda)} \frac{d}{d \lambda} \frac{\left\langle n\left|\partial_{\lambda}\right| 0\right\rangle}{E_{n}(\lambda)-E_{0}(\lambda)}+\ldots\right] \mathrm{e}^{i\left(\Theta_{n}(\lambda)-\Theta_{0}(\lambda)\right)}\right|_{\lambda_{i}} ^{\lambda_{f}}
\end{aligned}
$$

where $\Theta_{n}(t)=\int_{0}^{t} E_{n}(\tau) d \tau$ is the dynamical phase (in general one should also add the Berry phase term) and $E_{n}$ is the eigenenergy of the state $|n\rangle$. We can anticipate that the critical point will dominate the dynamics so that only one of the limits $\lambda=\lambda_{i}$ (if $\lambda_{i}=0$ ) or $\lambda=\lambda_{f}$ (if $\lambda_{f}=0$ ) should dominate the transition amplitude. From this equation it is clear that the lowest non-vanishing time derivative of $\lambda(t)$ at the critical point dominates the asymptotics of the transition probability:

$$
\left|\alpha_{n}\right|^{2} \approx v^{2} \frac{\left|\left\langle n\left|\partial_{\lambda}\right| 0\right\rangle\right|^{2}}{\left(E_{n}(0)-E_{0}(0)\right)^{2 r}}=v^{2} \frac{|\langle n|V| 0\rangle|^{2}}{\left(E_{n}(0)-E_{0}(0)\right)^{2 r+2}},
$$


where all the matrix elements are evaluated at the quantum critical point. Therefore we find that the probability of excitations, defined with respect to the instantaneous ground state, scales as:

$$
P_{\mathrm{ex}}=\sum_{n \neq 0}\left|\alpha_{n}\right|^{2} \approx L^{d} v^{2} \chi_{2 r+2}(0),
$$

where

$$
\chi_{m}(\lambda)=\frac{1}{L^{d}} \sum_{n \neq 0} \frac{|\langle n|V| 0\rangle|^{2}}{\left(E_{n}(\lambda)-E_{0}(\lambda)\right)^{m}}
$$

is the generalized adiabatic susceptibility of order $m$. For $m=2(r=0) \chi_{2}=\chi_{f}$, where $\chi_{f}$ is the fidelity susceptibility:

$$
\chi_{f}=\frac{1}{L^{d}} \sum_{n \neq 0}\left|\left\langle 0\left|\partial_{\lambda}\right| n\right\rangle\right|^{2}=\frac{1}{L^{d}} \sum_{n \neq 0} \frac{|\langle 0|V| n\rangle|^{2}}{\left(E_{n}(\lambda)-E_{0}(\lambda)\right)^{2}} .
$$

Thus $\chi_{f}$ describes the system's response to a sudden quench (see Refs. [45] and [52]). For $m=4(r=1)$ the linear adiabatic susceptibility $\chi_{4}$ describes the response to a linear quench and so on. Let us note that the scaling dimension of the susceptibilities $\chi_{m}(\lambda)$ is related to the scaling dimension of the fidelity susceptibility via: $\operatorname{dim}\left[\chi_{m}(\lambda)\right]=\operatorname{dim}\left[\chi_{f}(\lambda)\right]-z(m-2)=d-2 / \nu-z(m-2)$. If $\operatorname{dim}\left[\chi_{m}(\lambda)\right]$ becomes negative $(d \nu<2+z \nu(m-2))$ then the susceptibility $\chi_{m}(\lambda)$ diverges at the quantum critical point. Since $\operatorname{dim}[\lambda]=1 / \nu$ the asymptotical behavior of $\chi_{m}(\lambda)$ at small $\lambda$ is:

$$
\chi_{m}(\lambda) \sim \frac{1}{|\lambda|^{2-d \nu+z \nu(m-2)}} .
$$

In finite size systems this divergence is cutoff by the system size $L$ when the correlation length $\xi(\lambda) \sim 1 /|\lambda|^{\nu}$ becomes comparable to $L$. Thus exactly at the quantum critical point the generalized adiabatic susceptibility scales as

$$
\chi_{m}(0) \sim L^{2 / \nu+z(m-2)-d} .
$$

For $m=2$ this scaling agrees with that of the fidelity susceptibility [53, 54]. If the scaling dimension of $\chi_{m}$ is positive then this generically indicates that this susceptibility has a cusp singularity, meaning that the asymptotics (9) and (10) become subleading.

Combining Eqs. (6) and (10) we find that if the condition $d \nu \leq 2(1+z \nu r)$ is fulfilled, then $P_{\mathrm{ex}}$ has the following asymptotic behavior in the adiabatic limit:

$$
P_{\mathrm{ex}} \approx v^{2} L^{2 / \nu+2 z r} .
$$

Since the probability $P_{\text {ex }}$ must always be smaller than one, this scaling must be valid only for quenches of small amplitude $|v| \ll 1 / L^{1 / \nu+z r}$. The physical meaning of this requirement is that the correlation length of the system associated with this velocity $\xi(v) \sim 1 /|v|^{\frac{\nu}{1+z r \nu}}$ must be big compared to the system size $\xi \gg L$. Physically the length scale $\xi(v)$ characterizes the crossover in the response of the system from the sudden to the adiabatic regimes similar to the Kibble-Zurek mechanism 32, 33]. I.e. for excitations with characteristic size (e.g. wavelength) shorter than $\xi(v)$ the quenching process looks adiabatic, while for excitations with size larger than $\xi(v)$ it looks sudden. For linear quenches $\xi(v) \sim 1 /|v|^{\frac{\nu}{1+z \nu}}$ indeed reproduces the characteristic Kibble-Zurek length scale [32, 33].

Let us point that the crossover to the regime with $P_{\mathrm{ex}}(v) \approx 1$ occurs precisely when the characteristic length scale $\xi(v)$ becomes of the order of the system size. This can be explained using a simple symmetry argument, which is easier to understand for sudden quenches $r=0$. In this case the quench corresponds to projecting the ground state in the critical state on the new basis. The quantity $1-P_{\mathrm{ex}}$ defines the probability that the system remains in the ground state after the quench. But if $\xi(v)<L$ the new ground state has a well defined symmetry absent at the critical point. Thus the probability to remain in the ground state should be close to zero. Similar considerations apply to slow quenches, $r>0$. Thus for quenches of higher rate $|v| \gg 1 / L^{1 / \nu+z r}$ (but still slow compared to the other microscopic scales of the system) the probability of exciting the system is close to one and is no longer a good quantity to characterize the system response (like the overlap of two ground states becomes very close to zero and thus not very informative). We argued [45] that in this case the physically relevant and meaningful quantity to look at is the density of generated excitations $n_{\text {ex }}$ (quasiparticles if we are dealing with nearly integrable models). Indeed the physical reason why the global probability of excitation rapidly approaches unity is that exciting even a single quasiparticle makes the new state orthogonal to the initial state. Yet physically we anticipate that exciting a single quasiparticle in the system should not change drastically the properties of the system. In particular, it should not affect the probability to excite the next quasiparticle. Since we are typically dealing with few body operators by performing a quench we couple only to the many-body states characterized by few quasiparticles. Thus it was argued that the density of generated quasiparticles should scale as:

$$
n_{\mathrm{ex}} \sim v^{2} L^{2 / \nu-d+2 z r}
$$

for $|v| \ll 1 / L^{1 / \nu+z r}$ and

$$
n_{\mathrm{ex}} \sim|v|^{d \nu /(z \nu r+1)}
$$

in the opposite limit. Note that the second scaling has a much wider domain of applicability than the first one since there is no requirement on vanishing quench amplitude with the system size. In particular, for $r=1$ the result (13) agrees with the Kibble-Zurek scaling predicted earlier in Refs. [29, 30]. Also for nonlinear quenches $r>1$ the scaling (13) agrees with the results of Refs. [48, 49], 
noting that $v=(1 / \tau)^{r}$ in the notations of Ref. [48]. Let us also point out that, as we will illustrate here for the specific case of the sine-Gordon model (Sec. V), for $d \nu>2$ the nonanalytic asymptotic with $|v|$ or $L$ in the scaling for $n_{\text {ex }}$ and $P_{\text {ex }}$ survives but becomes subleading. The leading asymptotic is then analytic and it comes from the excitations of non-universal high energy states.

The density of excitations is a very simple and intuitive object, however it is not always well defined. Unless we deal with long lived quasiparticles or other e.g. topological excitations, in nonintegrable systems $n_{\mathrm{ex}}$ is not conserved in time after the quench because of various relaxation processes. For the same reason $n_{\mathrm{ex}}$ is not a readily observable quantity. Hence one needs to characterize the response of the system by other means. The two natural quantities, which can be defined for any Hamiltonian system, are the (diagonal) entropy [46] and the heat (or the non-adiabatic part of the energy, which is also the excess energy above the new ground state of the quenched Hamiltonian [4]]). Because both quantities are extensive, it is convenient to deal with their densities:

$$
\begin{aligned}
S_{d} & =-\frac{1}{L^{d}} \sum_{n}\left|\alpha_{n}\right|^{2} \log \left|\alpha_{n}\right|^{2}, \\
Q & =\frac{1}{L^{d}} \sum_{n}\left(E_{n}-E_{0}\right)\left|\alpha_{n}\right|^{2}=\sum_{n \neq 0} E_{n}\left|\alpha_{n}\right|^{2} .
\end{aligned}
$$

Here the index $n$ enumerates the many-body eigenstates of the Hamiltonian in the final state and $\left|\alpha_{n}\right|^{2}$, as written above in Eqs. (4) and (5)), is the probability of the transition to the $n$-state due to the quench. The scaling of the entropy is very similar (up to possible logarithmic corrections) to the one of $P_{\mathrm{ex}}$ and $n_{\mathrm{ex}}$. The advantage of the entropy over $P_{\mathrm{ex}}$ is that it is well behaved and meaningful for large amplitude quenches $|v| \gg 1 / L^{1 / \nu+z r}$, where $P_{\text {ex }} \rightarrow 1$. The scaling of the heat is different from that of $n_{\mathrm{ex}}$ because of the term $E_{n}-E_{0}$ in Eq. (15). If the quench ends at the critical point then one finds that the scaling of the heat is described by $\chi_{2 r+1}: Q \sim v^{2} \chi_{2 r+1}$ so that

$$
Q \sim v^{2} L^{2 / \nu-d+z(2 r-1)}
$$

for $|v| \ll 1 / L^{1 / \nu+z r}$ and

$$
Q \sim|v|^{(d+z) \nu /(z \nu r+1)}
$$

for $|v| \gg 1 / L^{1 / \nu+z r}$.

Let us note that the susceptibilities $\chi_{m}$ are directly related to the connected parts of the correlation functions $G_{\lambda}(\tau)=\langle V(\lambda, \tau) V(\lambda, 0)\rangle-\langle V(, \lambda, 0)\rangle^{2}$ by a simple generalization of the expressions obtained in Ref. [53]:

$$
\chi_{m}(\lambda)=\frac{1}{L^{d}(m-1) !} \int_{0}^{\infty} \tau^{m-1} G_{\lambda}(\tau) d \tau .
$$

For local perturbations $V=\sum_{x} V(x)$ it is obvious that if $G(\tau)$ decays sufficiently fast at large $\tau$ then $\chi_{m}$ is a non-negative number well defined in the thermodynamic limit $L \rightarrow \infty$. For the sine-Gordon model we wrote the explicit expressions of $\chi_{1}$ and $\chi_{2}$ in Eqs. (52) and (53). Using the Lehmann's representation of the correlation function we can define the generating functions for the susceptibilities $\chi_{m}(\lambda)$ :

$$
\begin{aligned}
I(\alpha) & =\frac{1}{L^{d}} \int_{0}^{\infty} G_{\lambda}(\tau) \mathrm{e}^{-\alpha \tau} d \tau \\
& =\frac{1}{L^{d}} \sum_{n \neq 0} \frac{|\langle n|V| 0\rangle|^{2}}{E_{n}(\lambda)-E_{0}(\lambda)+\alpha}
\end{aligned}
$$

such that

$$
I(\alpha)=\sum_{m}(-\alpha)^{m} \chi_{m}(\lambda) .
$$

This characteristic function clearly contains the information about the scalings laws of the quantities discussed in this work for all possible power law quenches.

Let us say a few words about the scaling in the regime where the relevant adiabatic susceptibility has only a cusp singularity. In this case the leading asymptotics of $\chi_{m}(\lambda)$ at $\lambda \rightarrow 0$ is given by a nonuniversal constant, which in turn defines the leading response of the system to the quench. Thus one can expect the scaling for the quantities $n_{\mathrm{ex}}, S_{d}, Q$ to be analytic in $v$. Instead of Eq. (12) we anticipate:

$$
n_{\mathrm{ex}} \sim v^{2} \chi_{2 r+2}(0)
$$

Similarly for the heat (in the case of a quench ending at the $\mathrm{QCP})$ we expect $Q \sim v^{2} \chi_{2 r+1}(0)$. As we will show below the non-analytic behavior of these quantities with $v$ survives, but becomes subleading and thus hardly identifiable either numerically or experimentally.

It is interesting to note that these scaling results extend to the situation of gapless systems even in the absence of the critical point. Thus if we are dealing with gapless bosonic theory then one simply needs to take the limit $\nu \rightarrow \infty$ in the expressions above. It is clear from Eqs. (9) and (10) that for $m>2$ the susceptibility $\chi_{m}$ can still diverge in low enough dimensions if $d<z(m-2)$. This divergence is actually the origin of the strong nonadiabaticity in low-dimensional gapless systems 55] (see also Ref. [56]). Thus the existence of the quantum criticality is not really needed to have a non-analytic scaling of various observables with $v$. Following Ref. [56] let us make the following observation. In the limit $\nu \rightarrow \infty$ in low dimensions the scaling of the three quantities $n_{\mathrm{ex}}, S_{d}$ and $Q$ is only sensitive to the quench time but not to the actual quenching protocol. For instance for $d<z(2 r-1)$ the scaling of the heat is $Q \sim|v|^{\frac{d+z}{z r}} \sim 1 / \tau^{\frac{d+z}{z}}$, where $\tau \sim\left(\lambda_{f} / v\right)^{1 / r}$ is the quench time required to change $\lambda$ from the initial value $\lambda_{i}=0$ to some fixed final value $\lambda_{f}$. Thus the scaling of $Q$ with $\tau$ is not-sensitive to the power $r$ with which the tuning parameter is quenched. On the other hand for $d>z(2 r-1)$ the situation is opposite. The scaling of the heat $Q \sim v^{2} \sim 1 / \tau^{2 r}$ is very sensitive to the power with which the coupling $\lambda$ is turned on 
(off). This suggests that the optimum quench protocol, which gives the smallest excitation level in the system for a given quench time $\tau$, corresponds to the crossover power $r=\frac{1}{2}\left(\frac{d}{z}+1\right)$. Higher powers of $r$ are to be avoided because of the growth of the prefactor with $r$ [49]. This argument extends the predictions of Ref. [49] to generic gapless systems.

\section{THE SINE-GORDON MODEL}

The SG-model is described by the Hamiltonian:

$$
H=\frac{1}{2} \int d x\left[\left(\partial_{x} \phi(x)\right)^{2}+(\Pi(x))^{2}-4 \lambda \cos (\beta \phi(x))\right],
$$

where $\phi(x)$ and $\Pi(x)$ are conjugated fields. Since this model is exactly solvable a great amount of facts is known about it. It is conventional to introduce the parameter $\xi$ [81]

$$
\xi=\frac{\beta^{2}}{8 \pi-\beta^{2}} .
$$

The spectrum of the SG Hamiltonian (22) depends on the value of $\beta$ (or $\xi$ ). We will be primarily interested in the regime of $0<\beta^{2}<8 \pi$ since in this range for a finite $\lambda$ the system is in a gapped phase, while for $\lambda=0$ it corresponds to the gapless Luttinger liquid. Therefore the system in this regime undergoes a quantum phase transition at $\lambda=0$. Furthermore for $4 \pi<\beta^{2}<8 \pi$ $(1<\xi<\infty)$, so called repulsive regime, the spectrum of the SG Hamiltonian consists of solitons and antisolitons. At fixed small $\lambda$ and $\beta^{2}=8 \pi$ the systems undergoes a Kosterlitz-Thouless transition to the Luttinger liquid regime, where the cosine term is irrelevant. The point $\beta^{2}=4 \pi(\xi=1)$, known as Toulouse point, maps to the free massive fermionic theory; at this point solitons and antisolitons correspond to particles and holes. For $0<\beta^{2}<4 \pi(0<\xi<1)$, so called attractive regime, the spectrum in addition to the solitons and antisolitons contains their bound states called breathers. The number of different types of breathers depends on the interaction parameter $\xi$ and is equal to the integer part of $1 / \xi$. We denote a breather by $B_{n}$ with $n=1,2, \ldots,[1 / \xi]$. In the small $\beta$ (or $\xi$ ) limit the SG model is well described by the gaussian approximation, where one expands the $\cos (\beta \phi)$ term in the Hamiltonian (22) to the quadratic order in $\phi$. In this limit there is only one massive excitation, corresponding to the lowest breather $B_{1}$. Solitons (kinks), antisolitons (antikinks), and breathers are massive particle-like excitations. The soliton and antisoliton mass in terms of the parameters of the Hamiltonian (22) was computed in Ref. [2]:

$$
M_{s}=\left(\frac{\pi \Gamma\left(\frac{1}{1+\xi}\right) \lambda}{\Gamma\left(\frac{\xi}{1+\xi}\right)}\right)^{(1+\xi) / 2} \frac{2 \Gamma\left(\frac{\xi}{2}\right)}{\sqrt{\pi} \Gamma\left(\frac{1+\xi}{2}\right)} .
$$

Breathers mass is related to the soliton mass via:

$$
M_{B_{n}}=2 M_{s} \sin \left(\frac{\pi \xi n}{2}\right) .
$$

Note that for weak interactions (small $\xi$ ) the lowest breather masses are approximately equidistant, $M_{B_{n}} \propto$ $n$, which suggests that these masses correspond to eigenenergies of a harmonic theory. There is a direct analogy between the breathers in the sine-Gordon model and the energy levels of a simple Josephson junction [8]. In the Josephson junction (which is described by the Hamiltonian (22) without the first gradient term) the energy levels also become approximately equidistant if the interaction (charging) energy is small. Let us note that because the SG theory is explicitly relativistic invariant the dynamical exponent $z$ is equal to 1 . The soliton mass $M_{s}$ identifies the characteristic energy scale of the system, therefore we expect for it a universal behavior around the critical point: $M_{s} \sim|\lambda|^{z \nu}$, that defines the critical exponent $\nu$ for this theory (from Eq. (24))

$$
\nu=\frac{1+\xi}{2}
$$

or $\nu=4 \pi /\left(8 \pi-\beta^{2}\right)$, which can be continuously varied between $1 / 2$ and $\infty$.

We will focus on sudden quenches of the parameter $\lambda$, $\lambda(t)=\lambda_{f} \Theta(t)$ and we will illustrate how the general scalings laws for the density of excited quasiparticles (Eqs. (12) and (13) ) can be obtained for this model. As we discussed in the previous section and in Ref. [45] these scaling laws are closely related to the scaling of the fidelity susceptibility $\chi_{f}$. This susceptibility can be expressed as an integral of the correlation functions. For the SG model the latter can be accessed from two limits: the infrared limit (IR) describing long distance properties, and the ultraviolet limit (UV) corresponding to short distances. The approach based on the form factors is well suited to analyze the former IR limit. To deal with the UV limit one can rely on the conformal perturbation theory, which is based on the fact that at short distances the SG model (as well as many other IR-massive models) is free and conformal (it effectively reduces to the Luttinger Liquid theory). These two approaches are complementary and can be used depending on the physics of interest. As we will show below for $d \nu<2$ (or $\nu<2$ since $d=1$ ) the former form factors approach reproduce the correct scalings of $P_{\mathrm{ex}}$ and $n_{\mathrm{ex}}$, while for $\nu>2$ one should use the conformal perturbation theory. Similar story is valid for heat but the IR and UV dominated domains are defined according to whether $(d+z) \nu=2 \nu$ is bigger or smaller than 2. In general, it is rather difficult to relate the results from the two formalisms: in order to get the UV physics from the form factors expansions one has to sum up the large (if not infinite) number of contributions (see Ref. [3] and the following Sec. IV and $\mathrm{V}$ ). The converse is also true. We will analyze both the UV and IR contributions to the excitation probability and show that their combination indeed reproduces the correct scaling asymptotics. 


\section{ANALYSIS OF THE LOW ENERGY EXCITATIONS BASED ON THE FORM FACTOR EXPANSION}

Let us start from the perturbative expression for the probability of exciting the system based on the adiabatic perturbation theory. Within the latter the transition amplitude to the state $|n\rangle$ is found as [50]

$$
\alpha_{n} \approx-\int_{0}^{\lambda_{f}} d \lambda\left\langle n\left|\partial_{\lambda}\right| 0\right\rangle
$$

If the matrix element $\left\langle n\left|\partial_{\lambda}\right| 0\right\rangle$ is not singular at the QCP then this expression reduces to the result of the first order of the conventional perturbation theory. The expression (27) has an advantage that it symmetrically treats both initial and the final values of the coupling $\lambda$ and gives convergent expressions even when the conventional perturbation theory breaks down. From Eq. (27) we find

$$
P_{\mathrm{ex}}\left(\lambda_{f}\right) \approx \sum_{n \neq 0} \int_{0}^{\lambda_{f}} \int_{0}^{\lambda_{f}} d \lambda_{1} d \lambda_{2}\left\langle 0\left|\partial_{\lambda_{1}}\right| n\right\rangle\left\langle n\left|\partial_{\lambda_{2}}\right| 0\right\rangle .
$$

In general one has to sum over all intermediate states $|n\rangle$. However, as we argued in [45], if $d \nu<2$ (i.e. $\xi<3$ in the SG model) then $P_{\text {ex }}$ is dominated by the low energy excitations. Since solitons and breathers are massive, i.e. gapped for $|\lambda|>0$, we can expect that the dominant contribution to Eq. (28) comes from single pairs of the lowest energy excitations (solitons and antisolitons for $\xi>1$ and $B_{1}$ breathers for $\xi<1$ ) with opposite momenta. Therefore the probability of exciting the system and the number of excited quasiparticles will become identical (up to a factor of two reflecting that one excited state creates two solitons or breathers). In this case we can identify the sum over the states $n$ as a sum over momenta $k$ and use:

$$
\begin{aligned}
\alpha_{k}\left(\lambda_{f}\right) & =-\int_{0}^{\lambda_{f}} d \lambda^{\prime}\left\langle k\left|\partial_{\lambda^{\prime}}\right| 0\right\rangle \\
& =2 \int_{0}^{\lambda_{f}} d \lambda^{\prime} \frac{\langle 0|\cos (\beta \phi)| k\rangle}{E_{k}\left(\lambda^{\prime}\right)-E_{0}\left(\lambda^{\prime}\right)},
\end{aligned}
$$

where $|k\rangle$ is a short-hand notation corresponding to the soliton-antisoliton (breather) pair with momenta $k$ and $-k$ and $E_{k}(\lambda)$ is the energy of such a pair. The expression for the density of excited quasiparticles $n_{\mathrm{ex}}$ is therefore:

$$
\begin{aligned}
n_{e x}\left(\lambda_{f}\right) & \approx \frac{1}{L} \sum_{k \neq 0}\left|\alpha_{k}\left(\lambda_{f}\right)\right|^{2}=\int \frac{d k}{2 \pi}\left|\int_{0}^{\lambda_{f}} d \lambda^{\prime}\left\langle 0\left|\partial_{\lambda^{\prime}}\right| k\right\rangle\right|^{2} \\
& =4 \int \frac{d k}{2 \pi}\left|\int_{0}^{\lambda_{f}} d \lambda^{\prime} \frac{\langle 0|\cos (\beta \phi)| k\rangle}{E_{k}\left(\lambda^{\prime}\right)-E_{0}\left(\lambda^{\prime}\right)}\right|^{2}
\end{aligned}
$$

Note that a factor of 2 should appear here, due to the fact the excited states correspond to pair of excitations, but it is exactly canceled since we are counting each pair twice integrating over all positive and negative momenta. Likewise in the same order of approximation one can obtain the expressions for the heat density $Q$ :

$$
Q\left(\lambda_{f}\right) \approx 4 \int \frac{d k}{2 \pi} E_{k}\left(\lambda_{f}\right)\left|\int_{0}^{\lambda_{f}} d \lambda^{\prime} \frac{\langle 0|\cos (\beta \phi)| k\rangle}{E_{k}\left(\lambda^{\prime}\right)-E_{0}\left(\lambda^{\prime}\right)}\right|^{2}
$$

and the entropy density:

$$
S_{d} \approx-\frac{1}{2} \int \frac{d k}{2 \pi}\left|\alpha_{k}\right|^{2} \ln \left|\alpha_{k}\right|^{2}
$$

There is an additional factor of $1 / 2$ in the expression for the entropy coming from the fact that particles are excited only in pairs and the contribution of each pair to the entropy (unlike $n_{\mathrm{ex}}$ ) is not doubled.

As we argued earlier (see also Ref. [45]) the advantage of using intensive quantities like $n_{\mathrm{ex}}, S_{d}, Q$ over $P_{\mathrm{ex}}$ is that the regime of validity of the perturbation theory for them is much bigger. Unlike for $P_{\mathrm{ex}}$ the quench amplitude is not required to vanish as some power of the system size. In Sec. VI we will explicitly illustrate this point for two exactly solvable limits of the SG model: $\xi=1$ and $\xi \ll 1$.

The adiabatic perturbation theory allows us to reduce the computation of the dynamical response to static correlation functions. The same is true if we are dealing with a slow processes where the coupling changes gradually in time. In this case (see Sec. II) there is an additional dynamical phase entering the transition amplitude. For example for linear quenches $\lambda(t)=v t$ instead of Eq. (29) we should use (see also Refs. [13, 50, 57]):

$$
\begin{aligned}
& \alpha_{k}(v)=\int_{0}^{\infty} d \lambda^{\prime} \frac{\langle 0|\cos (\beta \phi)| k\rangle}{E_{k}\left(\lambda^{\prime}\right)-E_{0}\left(\lambda^{\prime}\right)} \\
& \times \exp \left[\frac{i}{v} \int_{0}^{\lambda^{\prime}} d \lambda^{\prime \prime}\left(E_{k}\left(\lambda^{\prime \prime}\right)-E_{0}\left(\lambda^{\prime \prime}\right)\right)\right]
\end{aligned}
$$

in all expressions for $n_{\mathrm{ex}}, Q$ and $S_{d}$.

The matrix elements appearing in Eqs. (29) and (33) are related to the form factors of the operator $\cos (\beta \phi)$. In general the form factors represent the matrix element of a particular operator between the vacuum and the asymptotic states (eigenstates of the Bethe ansatz) created by the Zamolodchikov-Faddeev operators corresponding to solitons, antisolitons and breathers. Because the SG theory is Lorentz-invariant it is convenient to use the rapidity variable $\theta,-\infty<\theta<\infty$, which parametrizes the energy and momentum of the soliton (breather):

$$
E=M_{s, B_{n}} \cosh \theta, \quad k=M_{s, B_{n}} \sinh \theta .
$$

The eigenstates of the SG model can then be labeled by $\left|\theta_{n} \ldots \theta_{1}\right\rangle_{a_{n} \ldots a_{1}}$, where $a_{i}=\{s, \bar{s}, n=1 \ldots[1 / \xi]\}$ correspond to solitons, antisolitons, and breathers. The correlation functions of an arbitrary operator $\mathcal{O}(x, t)$ are 
written as an infinite series expansion in terms of all the asymptotic states [58]:

$$
\begin{aligned}
& \left\langle\mathcal{O}(x, t) \mathcal{O}^{\dagger}(0,0)\right\rangle=\sum_{n=0}^{\infty} \sum_{\left\{a_{i}\right\}} \int \prod_{i=1}^{n} \frac{d \theta_{i}}{(2 \pi)^{n} n !} \\
& \mathrm{e}^{i \sum_{j=1}^{n} k_{j} x-E_{j} t}\left|\left\langle 0|\mathcal{O}(0,0)| \theta_{n} \ldots \theta_{1}\right\rangle_{a_{n} \ldots a_{1}}\right|^{2} .
\end{aligned}
$$

The matrix elements entering Eqs. (29) and (33) are then explicitly related to the form factors of the operator $\cos (\beta \phi)$ in the two-particle asymptotic states:

$$
\left.\langle 0|\cos (\beta \phi)| k\rangle=\frac{1}{E(\theta)}\langle 0|\cos (\beta \phi)| \theta,-\theta\rangle\right\rangle_{a_{2}, a_{1}} .
$$

Here the additional factor of $1 / E(\theta)$ comes from the relativistic normalization of the eigenstates parametrized by $\theta,\left\langle\theta \mid \theta^{\prime}\right\rangle=\delta\left(\theta-\theta^{\prime}\right) /(2 \pi)$. While in Eq. (30) we used a different normalization $\left\langle k \mid k^{\prime}\right\rangle=\delta\left(k-k^{\prime}\right) /(2 \pi)$. Taking into account the relations (34) we indeed find that the normalization factor $1 /\left|d_{\theta} k(\theta)\right|=1 / E(\theta)$.

Let us discuss the two-soliton contribution to $n_{\text {ex }}$ first, which is expected to be dominant for $1 \leq \xi<3$. The corresponding form factors in the SG model are known in the literature [59, 60]:

$$
\begin{aligned}
& F_{\exp ( \pm i \beta \phi)}\left(\theta=\theta_{2}-\theta_{1}\right) \equiv\left\langle 0\left|e^{ \pm i \beta \phi}\right| \theta_{2}, \theta_{1}\right\rangle_{s \bar{s}} \\
& =\mathcal{G}_{\beta} G(\theta) \sum_{\sigma= \pm} \frac{\cosh (\theta / 2) \exp \left[\sigma \frac{\theta+i \pi}{2 \xi}\right]}{\sinh \left(\frac{\theta+i \pi}{\xi}\right)}
\end{aligned}
$$

where we kept only the relevant factors (those which depend on either $\theta$ or $\lambda_{f}$ ). We point out that the summation over $\sigma$ comes from considering both the possibilities for a pair of excitations (soliton-antisoliton and antisoliton-soliton). Also we write both signs in the exponent of the operator $e^{ \pm i \beta \phi}$ since these form factors are invariant with respect to the change of sign. Furthermore it is known that:

$$
\mathcal{G}_{\beta} \sim M_{s}^{2 \xi /(1+\xi)} .
$$

This result together with Eq. (36) implies that $\langle 0|\cos (\beta \phi)| k\rangle \sim\left|M_{s}\right|^{\frac{\xi-1}{\xi+1}} P(\theta) \sim\left|\lambda_{f}\right|^{\frac{\xi-1}{2}} P(\theta)$, where $P(\theta)$ is some function which only depends on $\theta$. Next we use this scaling of the matrix element in Eq. (30) for finding $n_{\mathrm{ex}}$. If the integral over $k$ (or equivalently over $\theta$ ) converges at small $k \sim M_{s}$ then we can perform the appropriate rescaling of the variables $\left(\lambda^{\prime} \rightarrow \lambda^{\prime} / \lambda_{f}\right.$ and $k \rightarrow k / M_{s}$ and send the upper limit of integration over $k$ to $\infty$. Then we immediately obtain the scaling $n_{\mathrm{ex}} \sim\left|M_{s}\right| \sim\left|\lambda_{f}\right|^{\nu}$, which agrees with the general prediction (13) in the case of a sudden quench. A similar analysis of the matrix elements leads to the correct scaling for slow quenches characterized by an arbitrary exponent $r$ (see Ref. [13] for linear quenches).

To analyze the convergence of the integrals we need to find the asymptotical behavior of the matrix element in Eq. (30) at large $\theta$. In order to do this, we look at the large $\theta$ behavior of the $\theta$-dependent piece in Eq. (37). In particular we find:

$$
G(2 \theta) \rightarrow \exp \left[ \pm \frac{1}{2}\left(\frac{1}{\xi}+1\right) \theta\right], \quad \theta \rightarrow \pm \infty,
$$

which could be derived from the infinite-product-gammafunctions representation of $G(\theta)$ 61] (or also, in a different way, see Eq. (61) in the book by F. Smirnov [59]). It is also straightforward to deduce the asymptotics of the remaining part of the form factor to finally get:

$$
F_{\cos (\beta \phi)}(2 \theta) \sim \exp \left[\theta\left(\frac{3}{2}-\frac{1}{2 \xi}\right)\right] .
$$

To find the matrix element $\langle 0|\cos (\beta \phi)| k\rangle$ ) we need to divide this asymptotical form by the soliton energy (see Eq. (36) $) E=M_{s} \cosh (\theta) \approx M_{s} \exp [\theta] / 2 \approx k$ at large $\theta$ so that

$\langle 0|\cos (\beta \phi)| k\rangle \sim M_{s}^{\frac{\xi-1}{\xi+1}} \exp \left[\frac{\theta}{2} \frac{\xi-1}{\xi}\right] \sim M_{s}^{\frac{\xi-1}{\xi+1}}\left(\frac{E}{M_{s}}\right)^{\frac{\xi-1}{2 \xi}}$.

This result correctly reproduces the scaling in the free fermionic limit of the theory $(\xi=1)$, which can be obtained by elementary methods (see Sec. VIC). Such scaling implies a logarithmic singularity at $\xi=1$ for the heat density in a sudden quench $Q \sim\left|\lambda_{f}\right|^{2} \log \left|\lambda_{f}\right|$ (see Eq. (31), which is equivalent to the log-divergence of the ground state energy. This divergence is expected because for $\xi=1$ we have $\nu=(1+\xi) / 2=1$, which corresponds to the crossover point between the scaling $Q \sim\left|\lambda_{f}\right|^{(d+z) \nu}$, expected for $\nu<2 /(d+z)$, and the quadratic scaling, expected for $\nu>2 /(d+z)$. For $\xi>1$ the integral over $k$ in Eq. (31) diverges at large $k$, i.e. the main contribution to the heat density comes from high energies (UV limit), where the lowest form factors expansion is not justified and we need to rely on the UV limit of the matrix elements discussed below in Sec. V] As for the other three quantities: $n_{\mathrm{ex}}, P_{\mathrm{ex}}$, and $S_{d}$ the scaling of the matrix element (41) together with Eqs. (30) and (32) suggests that $n_{\mathrm{ex}} \sim\left|\lambda_{f}\right|^{d \nu}=\left|\lambda_{f}\right|^{\frac{1+\xi}{2}}$ holds for the whole soliton region $1 \leq \xi<\infty$ and there is no indication of crossover to the quadratic scaling at $\xi=3$ (corresponding to $\nu=2$ ). This result is however misleading because it comes from the fact that the lowest order form factor expansion does not correctly reproduce the ultra-violet asymptotics of the matrix elements. As we mentioned above to get the right UV limit one needs to perform an infinite re-summation of the form factors which implies that the sum in Eq. (28) should be taken over multiple soliton states. This problem is well known in the equilibrium analysis of the UV asymptotics of the static correlation functions [62]. In the next section we will show how the power two emerges from analyzing Eq. (28) using the conformal perturbation theory.

Likewise one can analyze the scaling of the matrix elements in the breather region $(\xi<1)$. Since we have $\nu=(1+\xi) / 2<1$ we expect that the scaling of all the 
quantities we are interested in $Q, P_{\mathrm{ex}}, n_{\mathrm{ex}}, S_{d}$ is dominated by low energies where the lowest form factor expansion is justified. So for $\xi<1$ we need to repeat the analysis above with the only difference that the states $|k\rangle$ in Eq. (29) denote pairs of breather states with opposite momenta. The form factors for breathers have a different scaling form than those for solitons. With the operator $\cos (\beta \phi)$ it is possible to have non-zero form factors of a single breather $B_{n}$ of the form $\left\langle 0|\cos (\beta \phi)| B_{n}\right\rangle$ if $n$ is even. Those form factors do not depend on the rapidity at all (see 63] for more details). The first $\theta$ dependent contribution into Eq. (28) comes from exciting two $B_{1}$ breathers. These breathers are the only addition to the solitons in the spectrum for $1 / 2 \leq \xi<1$ and they are also the only surviving excitations in the noninteracting bosonic limit of the SG model $\xi \rightarrow 0$. Therefore here we will concentrate only on the scaling analysis of $n_{\text {ex }}$ and other observables coming from exciting pairs of $B_{1}$ breathers with opposite momenta [82]. The matrix element describing this process is found through the corresponding form factors which were computed in Ref. 63] using bootstrap, the procedure of fusion of several breathers [64]:

$$
\begin{aligned}
\langle 0|\cos (\beta \phi)| k\rangle & =\frac{1}{E(\theta)}\left\langle 0|\cos \beta \phi| B_{1}(\theta) B_{1}(-\theta)\right\rangle \\
& =\frac{1}{2 M_{s} \cosh \theta} \mathcal{G}_{\beta} \gamma^{2} \frac{\sin (\pi \xi)}{\pi \xi} R(2 \theta),(42)
\end{aligned}
$$

where

$$
\gamma=2 \cos \left[\frac{\pi \xi}{2}\right] \sqrt{2 \sin \left[\frac{\pi \xi}{2}\right]} \exp \left[-\int_{0}^{\pi \xi} \frac{d t}{2 \pi} \frac{t}{\sin t}\right]
$$

and

$$
R(\theta)=\exp \left[4 \int_{0}^{\infty} \frac{d t}{t} \frac{\sinh (t) \sinh (t \xi) \sinh (t(1+\xi))}{\sinh ^{2}(2 t)}\right] \exp \left[8 \int_{0}^{\infty} \frac{d t}{t} \frac{\sinh (t) \sinh (t \xi) \sinh (t(1+\xi))}{\sinh ^{2}(2 t)} \sinh ^{2} t\left(1-i \frac{\theta}{\pi}\right)\right]
$$

Since the dependence of the matrix element (42) on $M_{s}$ (and hence on $\lambda_{f}$ ) is again found via the function $\mathcal{G}_{\beta}$, we conclude that the scaling of $n_{\text {ex }}$ (as well as $Q, S_{d}$ and $\left.P_{\text {ex }}\right)$ on $\lambda_{f}$ is the same as in the repulsive regime, e.g. $n_{\mathrm{ex}} \sim\left|\lambda_{f}\right|^{(1+\xi) / 2}$. We only need to verify that the integrals over momenta $k$ converge at large $k$. In order to do this we need to know the asymptotics of the function $R(2 \theta)$ at large $\theta$. The scaling at large rapidity for the function $R(\theta)$ (defined in [60]) is $R(\theta) \sim F_{B_{1}}^{2}$, which does not depend on $\theta$. This saturation, which is the consequence of bootstrap, is actually true for higher order form factors as well. For example $\left\langle 0|\cos \beta \phi| B_{2}(\theta) B_{2}(-\theta)\right\rangle \sim$ $\left(F_{B_{2}}\right)^{2}$. On the other hand, because of the bootstrap relation, this should be proportional to $\left\langle 0|\cos \beta \phi| B_{4}\right\rangle$ which is a finite number. The one-breather form factors of the type $\left\langle 0\left|e^{i \beta \phi}\right| B_{n}(0)\right\rangle \equiv F_{B_{n}}$ can be computed from the residue of the soliton-antisoliton form factors at points $\left.\theta_{n}=i \pi(1-n \xi)\right)$, or, equivalently from the bootstrap (the definition of $F_{B_{n}}$ can be found in $[63]$ ). For example, the breather $B_{2}$ is a bound state of two breathers $B_{1}$. Using this bootstrap procedure we can find

$$
F_{B_{n}}^{\exp (i \beta \phi)}=\frac{\mathcal{G}_{\beta} \sqrt{2} \cot \left(\frac{\pi \xi}{2}\right) \sin (\pi n \xi) \exp [I(-\theta)] e^{\frac{i \pi n}{2}}}{\sqrt{\cot \left(\frac{\pi \xi n}{2}\right) \prod_{s=1}^{n-1} \cot ^{2}\left(\frac{\pi \xi s}{2}\right)}}
$$

where

$$
I(\theta)=\int_{0}^{\infty} \frac{d t}{t} \frac{\sinh ^{2}\left[t\left(1-\frac{i \theta}{\pi}\right)\right] \sinh [t(\xi-1)]}{\sinh [2 t] \cosh [t] \sinh [t \xi]} .
$$

The saturation of the form factors implies that the matrix elements $\langle 0|\cos (\beta \phi)| k\rangle$ behave as $1 / E(k)$ at large $k$ ensuring the convergence of integrals in Eqs. (30), (31), and (32) and thus the validity of the universal scalings. In the next section using the conformal perturbation theory we will also show that there are no UV issues with these scaling relations, thus the lowest form factor expansion is indeed justified. We note that the saturation of the breather form factors can be also understood from the fact that $\cos (\beta \phi)$ corresponds to the trace of the stressenergy tensor, whereas breathers correspond to the field $\phi$ itself. This saturation is thus expected for all breather contributions corresponding to exciting more than a single pair of breathers. In Fig. 1 we show the dependence of the $B_{1} B_{1}$ and $B_{2} B_{2}$ breather form factors as a function of the rapidity. Both plots indicate clear saturation with $\theta$ at the value corresponding to the square of the single breather form factor.

\section{ANALYSIS OF THE HIGH ENERGY EXCITATIONS BASED ON THE FIDELITY SUSCEPTIBILITY AND THE CONFORMAL PERTURBATION THEORY}

The other approach for finding the scaling of $P_{\mathrm{ex}}$ and other quantities with the quench amplitude $\lambda_{f}$ is based on the analysis of the fidelity susceptibility $\chi_{f}$, which is in turn related to the correlation functions. From Eq. (6) we know that for sudden quenches of small amplitude we can write:

$$
P_{\mathrm{ex}}\left(\lambda_{f}\right) \approx L\left|\lambda_{f}\right|^{2} \chi_{f}\left(\lambda_{f}\right)
$$




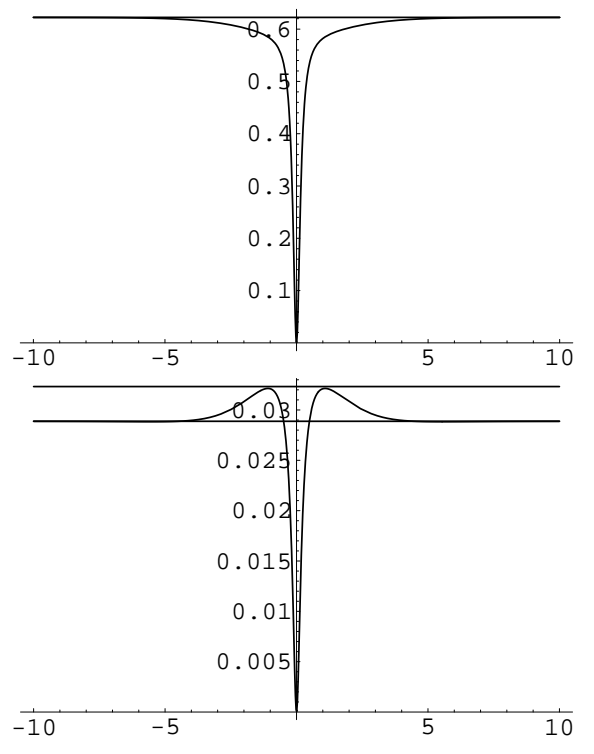

FIG. 1: $B_{1} B_{1}$ (top) and $B_{2} B_{2}$ (bottom) breather form factor for $\xi=1 / 41$ as a function of the rapidity $\theta$. The saturation lines are given by $\left(F_{B_{1}}\right)^{2}$ and $\left(F_{B_{2}}\right)^{2}$ respectively.

with $\chi_{f}$ defined in Eq. (8). A more accurate analysis based on the Cauchy-Schwartz inequality (see Sec. VII) shows that $P_{\mathrm{ex}}$ is bounded by the integral of $\chi_{f}$ as $P_{\mathrm{ex}}\left(\lambda_{f}\right) \leq L \lambda_{f} \int_{0}^{\lambda_{f}} d \lambda \chi_{f}(\lambda)$, still the scaling of $\lambda_{f} \int_{0}^{\lambda_{f}} \chi_{f}(\lambda) d \lambda$ and $\lambda_{f}^{2} \chi_{f}\left(\lambda_{f}\right)$ is the same for any powerlaw dependence of $\chi_{f}\left(\lambda_{f}\right)$, which we typically find near the critical point. Moreover in this section we will be primarily interested in the UV limit, corresponding to exciting high energy states, where Eq. (47) is justified by the conformal perturbation theory. Likewise one can obtain the expression for heat density:

$$
Q \approx\left|\lambda_{f}\right|^{2} \chi_{E}\left(\lambda_{f}\right)
$$

with :

$$
\chi_{E}(\lambda)=\frac{1}{L^{d}} \sum_{n \neq 0} \frac{|\langle 0|V| n\rangle|^{2}}{E_{n}(\lambda)-E_{0}(\lambda)} .
$$

The susceptibility $\chi_{E}$ is equal to the generalized adiabatic susceptibility of order one: $\chi_{E}=\chi_{1}$ according to Eq. (77).

For sudden quenches the wave function does not change during the quench. Hence the total energy after the quench is given by the expectation value of the new Hamiltonian taken with the initial wavefunction:

$$
E_{+}=\left\langle\Psi_{0}\left|H_{0}+\lambda_{f} V\right| \Psi_{0}\right\rangle,
$$

where $H_{0}$ is the free conformal Hamiltonian corresponding to the critical point and $V=-2 \int d x \cos (\beta \phi)$. In the conformal limit the ground state is invariant under the uniform phase rotation $\phi(x) \rightarrow \phi(x)+\phi_{0}$, where $\phi_{0}$ is an arbitrary phase. Using this symmetry the second term in Eq. (50) drops out and thus the total energy does not change during the quench. The heat is defined as the difference between the total energy and the adiabatic (ground state) energy of the instantaneous Hamiltonian $\left(E_{\mathrm{gs}}\left(\lambda_{f}\right)\right)$. Therefore we see that in our case the heat is equal by magnitude and opposite by sign to the change of the ground state energy: $Q=-\left(E_{\mathrm{gs}}\left(\lambda_{f}\right)-E_{\mathrm{gs}}(0)\right)$. One can recognize that Eq. (48) indeed gives (up to the sign) the second order perturbative correction to the ground state energy. As in the case of $P_{\mathrm{ex}}$, the perturbative expression is justified if we are interested in understanding the UV limit, where the energy denominator (see Eq. (49)) is large.

As we argued in Sec. I the susceptibilities $\chi_{E}$ and $\chi_{f}$ can be expressed through the integral of the connected part of the imaginary time correlation function $G(x, \tau)$ :

$$
\begin{aligned}
G(x, \tau) & =\langle 0|\cos (\beta \phi(x, \tau)) \cos (\beta \phi(0,0))| 0\rangle \\
& -\langle 0|\cos (\beta \phi(0,0))| 0\rangle^{2} .
\end{aligned}
$$

Namely, for a translationally invariant system we have:

$$
\begin{aligned}
& \chi_{E}=\int_{0}^{\infty} d \tau \int_{0}^{L} d x G(x, \tau), \\
& \chi_{f}=\int_{0}^{\infty} d \tau \int_{0}^{L} d x \tau G(x, \tau) .
\end{aligned}
$$

Let us point out that $G(x, \tau)$ is a monotonically decreasing non-negative function of $r=\sqrt{x^{2}+\tau^{2}}$ vanishing in the limit $r \rightarrow \infty$. The non-negativity of $\int d x G(x, \tau)$ follows from the Lehmann's representation:

$$
\int_{0}^{L} d x G(x, \tau)=\frac{1}{L} \sum_{n \neq 0}|\langle 0|V| n\rangle|^{2} \exp \left[-\tau\left(E_{n}-E_{0}\right)\right] .
$$

The relativistic invariance of the SG model also implies the non-negativity of $G(x, \tau)$ itself.

The scaling of $P_{\mathrm{ex}}$ and $Q$ with $\lambda_{f}$, at small $\lambda_{f}$, is thus related to the small $\lambda_{f}$ behavior of the integrals of $G(x, \tau)$. The long distance behavior of the correlations functions at scales $r \gg M_{s}^{-1}$ is given by the form factor expansion and can be analyzed by the methods of the previous section, leading to essentially the same predictions. In particular, because at any finite $\lambda_{f}$ the spectrum of the SG model is gapped, $G(x, \tau)$ exponentially decays on a correlation length scales $r \approx \zeta \sim 1 / M_{s} \sim 1 /\left|\lambda_{f}\right|^{(1+\xi) / 2}$. The scaling dimension of the operator $\cos (\beta \phi(x, \tau))$, entering the correlation function, is $\beta^{2} / 4 \pi=2 \xi /(1+\xi)$, implying that the scaling dimension of $G$ is twice that. Thus we can estimate the contribution to the scaling of $\chi_{f}$ coming from $\tau, x \sim \zeta$ as $\chi_{f} \sim \zeta^{3} / \zeta^{4 \xi /(1+\xi)} \sim$ $\left|\lambda_{f}\right|^{(1+\xi) / 2-2} \sim\left|\lambda_{f}\right|^{\nu-2}$. This is precisely the scaling we obtained from the form factors expansion.

The primary goal of this section is to analyze the UV (short distance) limit where the form factor expansion in the lowest order does not reproduce the correct scaling behavior, as we pointed out in Sec. [IV] For the contribution to the susceptibilities and thus to $P_{\mathrm{ex}}$ and $Q$ (as well 
as to $n_{\mathrm{ex}}$ and $\left.S_{d}\right)$ coming from $r \ll \zeta \sim M_{s}^{-1}$ one can use the cosine term as a small perturbation over the free (or conformal) limit. We note that by the uncertainty principle the short distances correspond to exciting states with high energies (and momenta) $E_{n} \gg M_{s}(k \gg 1 / \zeta)$. In turn the short distance behavior of the correlation functions is governed by power-law asymptotics with the exponents given by the scaling (conformal) dimensions of the operator $\cos (\beta \phi)$, (which is $\beta^{2} / 4 \pi=2 \xi /(\xi+1)$ as previously said). Thus in Euclidean notations:

$$
G(x, \tau) \sim \frac{1}{\left(x^{2}+\tau^{2}\right)^{\beta^{2} / 4 \pi}} .
$$

While this result is natural, it is somewhat nontrivial to obtain from the form factor perturbation theory since it requires an infinite re-summation of the form factors as it is nicely demonstrated in Refs. [3, 65]. So we can conclude that in order to correctly reproduce the high energy contribution to $P_{\mathrm{ex}}$ and $Q$ it is not sufficient to restrict the excited states in Eqs. (8), (28) and (49) to simply pairs of solitons and antisolitons with opposite momenta. It appears that only in the free fermionic point $\xi=1$ the lowest soliton-antisoliton contribution to $G(x, \tau)$ correctly predicts the exponent in Eq. (55). We believe that in general in our out-of-equilibrium setup it is possible to use a combination of some RG-type computations with the form factors based approach similar to the one in Ref. [66].

From Eqs. (52) and (53), we see that at the critical point $\chi_{E}$ has IR divergence at $\beta^{2} \leq 4 \pi$ corresponding to $\xi<1$ and $\chi_{f}$ has such divergence at $\beta^{2} \leq 6 \pi$ corresponding to $\xi<3$. By now we understand that this divergence simply implies that the scaling of $Q\left(\lambda_{f}\right)$ and $P_{\mathrm{ex}}\left(\lambda_{f}\right)$ is non-analytic in $\lambda_{f}$ or the system size in these domains. This divergence also indicates that the main contribution to the susceptibilities, and thus to the heat and the probability of exciting the system, comes from small energies $E \lesssim M_{s}$, where one can reliably use the lowest form factors expansion. Conversely for $\xi>1$ the susceptibility $\chi_{E}$ has a UV divergence, which is cutoff by the non-universal short distance regularization of the SG model. Similarly for $\xi>3$ the fidelity susceptibility $\chi_{f}$ is dominated by short distances or high energies. This implies that in these regimes of UV divergence, $\chi_{E}(\lambda)$ and $\chi_{f}(\lambda)$ approach non-universal constant values as $\lambda \rightarrow 0$ (note that these values are strictly positive because $G(x, \tau)$ is strictly positive). Therefore the leading asymptotical behavior for the heat becomes quadratic for $\xi>1: Q \sim\left|\lambda_{f}\right|^{2} \chi_{E}(0)$. Likewise the scaling for $P_{\mathrm{ex}}$ becomes extensive for $\xi>3: P_{\mathrm{ex}} \sim L \lambda_{f}^{2} \chi_{f}(0)$. This exactly agrees with the crossover to the quadratic scaling anticipated in the Sec. II] that occurs for $(d+z) \nu>2$ for $Q$ and for $d \nu>2$ for $P_{\mathrm{ex}}$. This crossover is directly analogous to the crossover between non-analytic and analytic regimes of scaling for the linear quenches in higher dimensions [55]. Similar crossover between perturbative and non-perturbative scalings at $\xi=3$ occurs for the density of excitations and the entropy.
While the short distance behavior of the correlation function given by the conformal limit (55) is intuitively anticipated, finding corrections to this scaling due to the finite value of the coupling $\lambda$ requires using conformal perturbation theory [67]. In the latter the starting point is the conformal field theory (free boson compactified on a circle of radius $R$ in our case). The Hilbert space then is the Verma module - representation space of the Virasoro algebra. The different basis states in this approach are labeled by two integers $(n, m)[83]$ corresponding to the eigenvalues $p_{ \pm}=n / R \pm m / 2 R$ of the momenta of the left/right movers $\varphi(z), \bar{\varphi}(\bar{z})$, with $\Phi(x, t)=\varphi(z)+\bar{\varphi}(\bar{z})$. The perturbed theory is constructed by adding vertex operators $V_{(n, m)}(z, \bar{z})=\exp \left[i\left(p_{+} \varphi(z)+p_{-} \bar{\varphi}(\bar{z})\right)\right]$ to the free theory. The scaling dimensions of the chiral parts of these vertex operators are $\Delta_{ \pm}=p_{ \pm}^{2} / 2$ so that the scaling dimension $h$ of $V_{(n, m)}$ is $h=\Delta_{+}+\Delta_{-}$. The sine-Gordon theory (22) is considered then as a perturbation of this free theory by the vertex operators with $n, m=\{ \pm 1,0\}$.

$$
\begin{aligned}
& H_{S G}=H_{C F T}+V, \\
& V=-2 \lambda_{f} \int_{0}^{L}\left(V_{(1,0)}(z, \bar{z})+V_{(-1,0)}(z, \bar{z})\right) d x \\
& \Phi(x, t)=\sqrt{4 \pi} \phi(x, t), \quad R=\sqrt{4 \pi} / \beta^{2} .
\end{aligned}
$$

The scaling dimension of $V$ is then $\beta^{2} / 4 \pi$. Since the Hilbert space is built by the action of the vertex operators on the vacuum state, $|n, m\rangle=V_{(n, m)}(0,0)|v a c\rangle$, (higher-energy states are built by the application of powers of the chiral components of the Kac-Moody currents $\partial \varphi$ to these states) the conformal perturbation theory is simply a perturbation theory in this space, and the effect of the perturbation is an expansion in powers of the $\left(M_{s} L\right)^{2-h}$, where $h=\beta^{2} / 4 \pi$ is the scaling dimension of the perturbation (so this expansion is in integer powers of $\lambda_{f}$ ). In Ref. 62] such expansion to the third power of $\lambda_{f}$ was applied to the correlation function. In particular, it was found that

$$
\left\langle 0\left|\mathrm{e}^{i \beta \phi(x, \tau)} \mathrm{e}^{-i \beta \phi(0,0)}\right| 0\right\rangle=\frac{1}{r^{\beta^{2} /(2 \pi)}}+C \lambda_{f}^{2} \frac{1}{r^{\beta^{2} / \pi-4}}+\ldots,
$$

where $r=\sqrt{x^{2}+\tau^{2}}$ and $C$ is a constant. In the repulsive regime $4 \pi<\beta^{2}<8 \pi$ where this expansion was derived the second term clearly decays slower at large $r$ than the first leading term indicating that the Taylor expansion in $\lambda_{f}$ can break down. This is of course anticipated because $\cos (\beta \phi)$ is the relevant perturbation. Conversely at small $r$ the first term corresponding to the conformal limit is dominant.

If we use this expansion to compute $\chi_{E}$ according to Eq. (52) then as we discussed above for $\beta^{2}<4 \pi$ (or $\xi<1$ ) the first term gives IR divergence which should be fixed by using the proper long distance asymptotics of the correlation function. On the other hand for $4 \pi<\beta^{2}<6 \pi$ $(1<\xi<3)$ the first term gives a finite constant contribution to $\chi_{E}$ while the second term proportional to $\lambda_{f}^{2}$ gives a divergence which again has to be fixed by the proper long distance asymptotics. For $3<\xi<5$ the second 
term gives a convergent contribution indicating that $\chi_{E}$ has analytic expansion in $\lambda_{f}$ up to $\lambda_{f}^{2}$ but the following term $\propto \lambda_{f}^{4}$ is expected to give a non-analytic contribution and so on. Thus from the conformal perturbation we can anticipate that the asymptotic expansion for the heat in powers of $\lambda$ has the following structure

$$
Q=\sum_{0<n<[(1+\xi) / 2]} \tilde{C}_{n} \lambda_{f}^{2 n}+\tilde{B}\left|\lambda_{f}\right|^{1+\xi}+o\left(\left|\lambda_{f}\right|^{1+\xi}\right)
$$

where $\tilde{C}_{n}$ are some non-universal constants which depend on the short distance cutoff and $\tilde{B}$ is a universal constant determined by the low energy (long distance) contribution to $\chi_{E}$ (and thus to the heat). At the special points $1+\xi=2 n$ the constant $\tilde{B}$ is expected to have logarithmic divergence.

Similar analysis can be done for $\chi_{f}$ using Eq. (53) and the expansion (58). Now we observe that the first conformal contribution diverges at $\beta^{2}<6 \pi(\xi<3)$. As before this divergence is cutoff by using the proper long distance asymptotics of $G(x, \tau)$ and results in the nonanalytic scaling of $\chi_{f}(\lambda)$. For $6 \pi<\beta^{2}<7 \pi(3<\xi<7)$ the first term in Eq. (58) gives a constant contribution to $\chi_{f}$, while the second term diverges and thus results in a non-analytic contribution. For $7<\xi<11$ we see that both the first and the second terms in (58) give convergent contribution to $\chi_{f}$, but we can anticipate that the third term gives a non-analytic correction and so on. We can therefore anticipate the scaling for the probability of exciting the system:

$P_{\mathrm{ex}}=L \sum_{n=1}^{(1+\xi) / 4} \breve{C}_{n} \lambda_{f}^{2 n}+\breve{B} \lambda_{f}^{2} L^{4 /(1+\xi)}+o\left(\left|\lambda_{f}\right|^{2} L^{4 /(1+\xi)}\right)$

where as before $\breve{C}_{n}$ are non-universal constants and $\breve{B}$ is universal and also expected to have log-divergence at the special points $1+\xi=4 n$. While we can expect a similar structure of the expansion for $n_{\mathrm{ex}}$ and $S_{d}$, it is harder to analyze these quantities in the UV regime because they do not have an explicit representation through the correlation functions. We simply note that the crossover to the leading quadratic scaling for these quantities when $\xi>3$ (with probable log corrections for the entropy) is expected on general grounds as a manifestation of the validity of usual perturbation theory applicable to the high energy excitations, where the probability of the transition to be excited scales as $\left|\alpha_{n}\right|^{2} \sim \lambda_{f}^{2}$. Since the low energy excitations coming from creating soliton-antisoliton pairs give $n_{\mathrm{ex}}, S_{d} \sim\left|\lambda_{f}\right|^{(1+\xi) / 2}$ we expect that the high energy excitations will dominate precisely when $(1+\xi) / 2>2$.

One can actually analyze the energy of an arbitrary state $|\Psi\rangle$, obtained by acting with the operator $\Psi$ on the vacuum $|\Psi\rangle=\Psi|0\rangle$, as a function of $\lambda_{f}$ using the nonlinear integration equation approach [68, 69]. The corresponding expansion in powers of $\lambda_{f}$ reads:

$$
\frac{E_{\Psi}}{M_{s}}=-\frac{\pi c}{6 l}+\frac{2 \pi h_{\Psi}}{l}+B l+\sum_{n=1}^{\infty} C_{n}(\Psi) l^{n\left(2-h_{\Psi}\right)}
$$

where $l=M_{s} L, B=-\tan (\pi \xi / 2) / 4$ is the bulk energy in the thermodynamic limit, $c$ is the central charge (equal to 1 in our case), $C_{n}(\Psi)$ are non-universal constants. The bulk energy becomes infinite at the point where $\xi$ is an odd integer. At this points there is a value of $n$ for which $n(2-h)=1$, i.e. $\beta^{2}=8 \pi(1-1 /(2 n))$ and $C_{n} \rightarrow \infty$. The infinite contributions from the bulk part and from the " $\mathrm{C}$ part" cancel giving the logarithmic singularity proportional to $l \log (l)$. These expectations are consistent with the findings of Refs. [68], [69]. In particular, it was found that at the special points $\beta^{2}=8 \pi(1-1 / 2 n)$, corresponding to $\xi=2 n-1$, the expression for the ground state energy gives logarithmic singularities. Exactly at these points the short distance expansion method of Konik and LeClair [62] gives the divergency of the $n$-th order of the perturbative expansion. Noting that for sudden quenches the heat is related to the ground state energy and thus to $\chi_{E}$ we see that the expansion (61) is consistent with the asymptotical form (59) suggested above.

Note that in the attractive regime $\beta^{2}<4 \pi, \xi<1$ the UV asymptotics of the correlation functions always give long distance divergent contribution to both $\chi_{E}$ and $\chi_{f}$ indicating that the corresponding susceptibilities are determined by the IR region where the lowest form factors expansion is accurate. This confirms that the general scalings obtained in Ref. [45] (see also Sec. II) and verified in the previous section are justified in the whole breather region.

Let us point out that a very similar analysis can be extended to slow quenches with arbitrary exponent $r$ (see Eq. (2)). The validity of the perturbative quadratic and extensive scaling of $P_{\mathrm{ex}}$ is determined by the convergence of the adiabatic susceptibility of order $2 r+2$ (see Eqs. (6) and (7)). In particular, for linear quenches, the relevant susceptibility $\chi_{4}(\lambda)$ is always IR divergent in the regime of our interest, $0<\beta^{2}<8 \pi$, implying that the scaling of $P_{\text {ex }}$ in the case of linear adiabatic quenches in one dimension [13] remains non-analytic in the whole gapped regime of the SG-model and the form factors analysis in this range is always justified. Thus in order to analyze the crossover between analytic and nonanalytic regimes of scaling we need to consider the generalizations of the SG model to higher dimensions. This can be easily done in the two limits $\xi=1$ and $\xi \ll 1$ corresponding to free massive fermionic and bosonic theories respectively. We will discuss these generalizations and the crossovers in the next section.

\section{EXACTLY SOLVABLE CASES: FREE MASSIVE BOSONS AND FERMIONS}

In this section we will focus on two limits of the SG model where the dynamics can be analyzed exactly without the need to rely on the adiabatic perturbation theory or other approximations. These limits have also the advantage to have straightforward multi-dimensional generalization. First we consider the limit of $\beta \ll 1$. In 
this case the cosine term in the Hamiltonian (22) can be expanded to the quadratic order in the phase $\phi$ and the problem maps into the free scalar massive bosonic field theory. The quench process corresponds to a sudden increase of the mass starting from zero. The quench dynamics in this regime was recently considered in Ref. [7] from a different point of view, analyzing the correlation functions and the asymptotic steady state. The other solvable limit corresponds to $\beta=2 \sqrt{\pi}(\xi=1)$. For this value of $\beta$ the problem deals with hard-core bosons or non-interacting fermions. In the language of fermions the quench process corresponds to a sudden turn on of a commensurate periodic potential, which opens a gap at the fermi momentum. In the following two sections we will discuss these limits in detail, analyze the scalings of the density of quasiparticles, heat, and entropy and show how those results agree with the general scaling predictions of Sec. III For these solvable cases in Sec. VID we will be able also to extend the exact results to quenches at finite temperatures.

\section{A. Elementary derivation of the scaling relations using the adiabatic perturbation theory}

Before proceeding with the analysis of specific bosonic and fermionic models we show how the scaling relations and the crossover to the quadratic scaling can be understood if we are dealing with a free theory. This discussion goes along the lines with that of Ref. 29] for slow linear quenches. Using the general discussion of Sec. [I we find that within the adiabatic perturbation theory the amplitude for creating an excited state in a sudden quench is:

$$
\alpha_{n}\left(\lambda_{f}\right)=\int_{0}^{\lambda_{f}} d \lambda \frac{\langle n|V| 0\rangle}{E_{n}(\lambda)-E_{0}(\lambda)} .
$$

If we are dealing with a free spatially uniform Hamiltonian then the operator $V$ must be quadratic in the creation and annihilation operators and conserve the total momentum. Thus acting on the vacuum state $V$ can either leave it intact or create a pair of quasiparticles with opposite momenta. Hence all the intermediate states can be characterized by the momentum label $q$. Thus the total probability to excite the system is:

$$
P_{\mathrm{ex}}=1-\prod_{q>0}\left(1-p_{\mathrm{ex}}(q)\right),
$$

where the product is taken over different pairs of twoparticle states with opposite momenta and $p_{\text {ex }}(q)=$ $\left|\alpha_{q}\left(\lambda_{f}\right)\right|^{2}$ is the probability to create the pair. In the limit when $\sum_{q>0} p_{\text {ex }}(q) \ll 1$ the product can be approximated by the sum and we have:

$$
P_{\mathrm{ex}} \approx \sum_{q>0} p_{\mathrm{ex}}(q)
$$

Note that since each term in the sum corresponds to a pair of quasiparticles the number of excited quasiparticles is given by the same expression above multiplied by a factor of two, which can be absorbed into the sum by summing over all positive and negative momentum states. Thus

$$
n_{\mathrm{ex}} \approx \frac{1}{L^{d}} \sum_{q}\left|\int_{0}^{\lambda_{f}} d \lambda \frac{\langle q|V| 0\rangle}{E_{q}(\lambda)-E_{0}(\lambda)}\right|^{2},
$$

where $|q\rangle$ is a short-hand notation for the state with two excited quasiparticles with momenta $q$ and $-q$ and $E_{q}(\lambda)$ is the corresponding excitation energy of the pair. Let us analyze the expression above using the scaling arguments (see also Refs. [29, 50, 57]). Near the critical point we expect that $E_{q}(\lambda)-E_{0}(\lambda)=|\lambda|^{z \nu} F\left(q / \lambda^{\nu}\right)$, with $F(x)$ a scaling function. In the limit $x \gg 1$ we must have $F(x) \sim$ $x^{z}$ to ensure the scale invariance at the critical point. Similarly we write the scaling of the matrix element:

$$
\frac{\langle q|V| 0\rangle}{E_{q}(\lambda)-E_{0}(\lambda)}=\frac{1}{|\lambda|} G\left(q /|\lambda|^{\nu}\right),
$$

where $G(x)$ is another scaling function. The same requirement of scale invariance at the critical point gives that $G(x) \sim 1 / x^{1 / \nu}$ at $x \gg 1$. Some examples where the scalings and asymptotics for the matrix element and the energy were explicitly confirmed can be found in Refs. [50, 55]. Using these scaling results it is straightforward to find the scaling for the density of excited quasiparticles. In particular, focusing on the limit $\left|\lambda_{f}\right| \gg$ $L^{-1 / \nu}$, where the summation over momenta can be replaced by the integration, and changing variables $q=$ $\left|\lambda_{f}\right|^{\nu} \eta$ and $\lambda=\lambda_{f} \xi$ in Eq. (65) we find that

$$
n_{\mathrm{ex}} \approx\left|\lambda_{f}\right|^{d \nu} \int \frac{d^{d} \eta}{(2 \pi)^{d}}\left|\int_{0}^{1} \frac{d \xi}{\xi} G\left(\eta /|\xi|^{\nu}\right)\right|^{2} .
$$

A similar expression is valid for the heat density:

$$
Q \approx\left|\lambda_{f}\right|^{(d+z) \nu} \int \frac{d^{d} \eta}{(2 \pi)^{d}} F(\eta)\left|\int_{0}^{1} \frac{d \xi}{\xi} G\left(\eta /|\xi|^{\nu}\right)\right|^{2} .
$$

We thus see that we are reproducing the scaling prediction of Sec. III Eqs. (13) and (17), for sudden quenches $(r=0)$ as long as the limits of the integration over $\eta$ can be extended to infinity. This is only justified if the integral over $\eta$ converges at large $\eta$. Using the asymptotical expression for the scaling functions $G$ and $F$ we see that this is the case for $d \nu<2$ if we are analyzing $n_{\mathrm{ex}}$ and for $(d+z) \nu<2$ if we are analyzing the heat. Alternatively the integrals over the momenta are dominated by the high energy cutoff and the scalings of the corresponding quantities become quadratic with $\lambda_{f}$, in accord with the predictions of Sec. III A similar analysis shows that the adiabatic perturbation theory reproduces the correct scaling also for the entropy density $S_{d} \sim\left|\lambda_{f}\right|^{d \nu}$ when $d \nu<2$. The same approach confirms the correct scaling of all these quantities for small quench amplitudes $\left|\lambda_{f}\right| \ll 1 / L^{1 / \nu}$, e.g. $n_{\mathrm{ex}}, P_{\mathrm{ex}} \sim\left|\lambda_{f}\right|^{2} L^{2 / \nu-d}$. The 
easiest way to see this is from matching the quadratic scaling and the universal scaling when the correlations length $\xi \sim 1 /\left|\lambda_{f}\right|^{\nu}$ becomes of the order of the system size. In the next two sections we will explicitly show how these crossovers emerge from the two free limits of the SG model.

\section{B. Free massive bosons}

Consider the SG Hamiltonian (22) when $\beta \rightarrow 0$. In this limit we can Taylor expand the cosine term up to the quadratic order in the field $\phi$ and obtain a quadratic Hamiltonian, that in the Fourier space has the form:

$$
H=\frac{1}{2} \sum_{q}\left|\Pi_{q}\right|^{2}+\kappa_{q}(\lambda)\left|\phi_{q}\right|^{2}
$$

with $\kappa_{q}(\lambda)=q^{2}+2 \lambda(t) \beta^{2}$ and $\Pi_{q}^{\dagger}, \phi_{q}^{\dagger}=\Pi_{-q}, \phi_{-q}$. We point out that here as in Eq. (22) we have set $\hbar v_{s}=1$, with $v_{s}$ being the sound velocity. For each momentum $q$ the fields $\Pi_{q}^{\dagger}$ and $\phi_{q}$ are conjugates, $\left[\Pi_{q}^{\dagger}, \phi_{q^{\prime}}\right]=i \delta_{q, q^{\prime}}$. Therefore we are dealing with a sum of independent harmonic oscillators with a time-dependent compressibility $\kappa$ (or the inverse mass). The wave function for this timedependent Hamiltonian can be found exactly, for instance in Refs. 50, 55] this was done for the case of an adiabaticlinear quench $(\lambda(t)=v t)$. Here we focus on the case of a sudden quench and show that the exact results agree with the general scaling predictions that have been presented in the Sec II. Furthermore the Hamiltonian (69) can be easily extended to higher dimensions and thus we are able to see how the crossover to the quadratic scalings and the usual perturbation theory emerges as the corresponding exponents $d \nu$ or $(d+z) \nu$ exceed two. We note that in this limit of the SG model $\nu=1 / 2$ and $z=1$. For the free theory these exponents remain the same in all dimensions.

From an experimental point of view this limit can be realized in the case of merging (coupling through tunneling) two one-dimensional Bose gases; indeed as it has been pointed out in [13] the SG hamiltonian (22) describes the merging process through the identification of $\beta=\sqrt{2 \pi / K}$, with $K$ the Luttinger parameter for each Bose gas, and $\lambda(t)$ proportional to the tunneling strength between the two tubes. In the limit of weakly interacting gases $K \rightarrow \infty$, hence $\beta \rightarrow 0$ and the process is described by the Hamiltonian (69).

Let us consider a sudden quench in the Hamiltonian (69) where $\lambda$ is changed in time as: $\lambda(t)=\lambda_{f} \Theta(t)$, where $\Theta(t)$ is the step function. It is convenient to switch to the representation in terms of the creation and annihilation operators $a^{\dagger}$ and $a$, such that $\left[a, a^{\dagger}\right]=1$, in order to work with the number-state vectors: $|n\rangle=\left(a^{\dagger}\right)^{n} / \sqrt{n !}|0\rangle$. In particular, for a fixed $\lambda$ those operators are:

$$
\begin{aligned}
& a_{q}(\lambda)=\frac{1}{(4 \kappa(\lambda))^{\frac{1}{4}}}\left(\Pi_{q}+i \sqrt{\kappa_{q}(\lambda)} \phi_{q}\right), \\
& a_{q}^{\dagger}(\lambda)=\frac{1}{(4 \kappa(\lambda))^{\frac{1}{4}}}\left(\Pi_{q}^{\dagger}-i \sqrt{\kappa_{q}(\lambda)} \phi_{q}^{\dagger}\right),
\end{aligned}
$$

for each mode $q$, such that the Hamiltonian in this basis becomes: $H=\sum_{q} \sqrt{\kappa(\lambda)}\left(a_{q}^{\dagger}(\lambda) a_{q}(\lambda)+1 / 2\right)$. For uniform quenches all $q$-modes are independent from each other and the dynamics of the system is factorizable. We are interested in evaluating the effects of the sudden quench on the population of the excited levels, therefore we want to express the initial ground state $\left|0_{q}\right\rangle_{0}$ before the quench $(\lambda=0)$ in terms of the eigenstates $\left\{\left|n_{q}\right\rangle_{\lambda_{f}}\right\}$ of the final Hamiltonian $\left(\lambda=\lambda_{f}\right)$ :

$$
\left|0_{q}\right\rangle_{0}=\sum_{n} c_{2 n}(q)\left|2 n_{q}\right\rangle_{\lambda_{f}}
$$

where we used the fact that only even modes are excited in the quench process because of the parity conservation. The coefficients $c_{2 n}(q)$ can be evaluated expressing the operator $a_{q}(0)$ in terms of $a_{q}^{\dagger}\left(\lambda_{f}\right)$ and $a_{q}\left(\lambda_{f}\right)$ :

$$
\begin{aligned}
c_{2 n}(q)= & (-1)^{n} \sqrt{\frac{(2 n-1) ! !}{(2 n) ! !}}\left(\frac{\sqrt{\kappa_{q}\left(\lambda_{f}\right)}-\sqrt{\kappa_{q}(0)}}{\sqrt{\kappa_{q}\left(\lambda_{f}\right)}+\sqrt{\kappa_{q}(0)}}\right)^{n} \\
& \times \sqrt{\frac{2\left(\kappa_{q}\left(\lambda_{f}\right) \kappa_{q}(0)\right)^{\frac{1}{4}}}{\sqrt{\kappa_{q}\left(\lambda_{f}\right)}+\sqrt{\kappa_{q}(0)}}} .
\end{aligned}
$$

One can check that these coefficients are properly normalized: $\sum_{n}\left|c_{2 n}(q)\right|^{2}=1$. Physically each state $\left|2 n_{q}\right\rangle$ represents $2 n_{q}$ quasiparticles with opposite and equal momenta $q$ and $-q$. Therefore the average number of excitations in each mode is $n_{\mathrm{ex}}(q)=\sum_{n} 2 n\left|c_{2 n}(q)\right|^{2}$. We can also formally define the probability that the mode with momentum $q$ is excited as:

$$
p_{\mathrm{ex}}(q)=\sum_{n \neq 0}\left|c_{2 n}(q)\right|^{2}
$$

The total probability of exciting the system $P_{\mathrm{ex}}$ is found as the complementary of the joint probability that no mode is excited after the quench:

$$
P_{\mathrm{ex}}=1-\prod_{q>0}\left|c_{0_{q}}\right|^{2}=1-\prod_{q>0}\left(1-p_{\mathrm{ex}}(q)\right) .
$$

In the limit $\sum_{q} p_{\mathrm{ex}}(q) \ll 1$ we see that $P_{\mathrm{ex}}(q) \approx$ $\sum_{q} p_{\mathrm{ex}}(q)$.

From Eq. (73) we find the condition $p_{\text {ex }}(q) \ll 1$ is satisfied if we impose:

$$
\frac{\sqrt{\kappa_{q}\left(\lambda_{f}\right)}-\sqrt{\kappa_{q}(0)}}{\sqrt{\kappa_{q}\left(\lambda_{f}\right)}+\sqrt{\kappa_{q}(0)}}=\frac{\sqrt{q^{2}+2 \lambda_{f} \beta^{2}}-q}{\sqrt{q^{2}+2 \lambda_{f} \beta^{2}}+q} \ll 1
$$

that implies $2 \lambda_{f} \beta^{2} \ll q^{2}$. In this limit we have $p_{\text {ex }}(q) \approx$ $\left|\lambda_{f}\right|^{2} \beta^{4} /\left(8 q^{4}\right)$. For a system with size $L$ the lowest 
nonzero momentum $q$ is $\frac{2 \pi}{L}$ and therefore the probabilities that $p_{\mathrm{ex}}(q) \ll 1$ for all $q$ is equivalent to:

$$
\lambda_{f} \ll \lambda_{s}=\frac{1}{2 \beta^{2}}\left(\frac{2 \pi}{L}\right)^{2} .
$$

It is obvious that this condition also ensures that $\sum_{q} p_{\mathrm{ex}}(q) \ll 1$. In this limit the total probability for the system to be excited is:

$$
P_{\mathrm{ex}} \approx \sum_{q} p_{\mathrm{ex}}(q) \approx \sum_{q} \frac{\beta^{4} \lambda_{f}^{2}}{8 q^{4}}=\frac{L^{4} \beta^{4} \lambda_{f}^{2}}{8(2 \pi)^{4}} \zeta(4)
$$

where $\zeta(k)$ is the Riemann's Zeta function. It is easy to check that the coefficient multiplying $L \lambda_{f}^{2}$ is precisely the fidelity susceptibility $\chi_{f}$ evaluated in the massless limit $\lambda=0$. The superlinear scaling of the excitation probability $P_{\mathrm{ex}} / L \sim L^{3}$ is consistent with the one anticipated in Ref. [45] (see also Sec. 【I): $\chi_{f}(0) \sim L^{-d+2 / \nu}=L^{3}$.

For quenches of larger amplitude, when $\lambda_{f}>\lambda_{s}$, as we already discussed in Sec I the probability of exciting the system $P_{\text {ex }}$ is almost unity. This indicates that $P_{\text {ex }}$ can not differentiate between different excited states. This comes from the fact that even if we excite a single pair of quasiparticles, the state becomes immediately orthogonal to the ground state and $P_{\mathrm{ex}}=1$. At the same time physically this state is almost indistinguishable from the ground state since a single pair of excited quasiparticles can not affect any thermodynamic properties of the system. Let us analyze instead that following object:

$$
\frac{\sum_{q} p_{\mathrm{ex}}(q)}{L} \approx \frac{1}{2 \pi} \int_{0}^{\infty} d q \sum_{n_{q} \neq 0}\left|c_{2 n(q)}\right|^{2} \approx 0.036 \sqrt{\lambda_{f} \beta^{2}}
$$

which represents the sum of probabilities of excitations of different momentum modes. In general such object can be defined first evaluating the two-particle density matrix in the momentum space and then finding probability that the corresponding mode is excited. To evaluate Eq. (79) we used the fact that the sum over momenta can be converted into the integral and for small amplitude quenches the limits of integration can be extended to $[0, \infty)$. The scaling dependence in (79) agrees with $\sim\left|\lambda_{f}\right|^{2} \chi_{f}\left(\lambda_{f}\right)=\left|\lambda_{f}\right|^{d \nu}$ and hence it illustrates that the fidelity susceptibility actually describes the behavior of $\sum_{q} p_{\mathrm{ex}}(q)$ rather than $P_{\mathrm{ex}}$. Only for infinitesimally small quenches $\left|\lambda_{f}\right| \ll\left|\lambda_{s}\right|$, when these two objects coincide, the fidelity susceptibility also describes the scaling of $P_{\mathrm{ex}}$. This point can be made even more explicit if we consider a quench starting and ending in the massive limit $\lambda_{i}>0$ and $\lambda_{f}=\lambda_{i}+\delta \lambda>0$. Then one can check that as long as $|\delta \lambda| \ll \lambda_{i}$ we have $\sum_{q} p_{\text {ex }}(q) \approx L(\delta \lambda)^{2} \chi_{f}\left(\lambda_{i}\right)$. At the same time a similar scaling for $P_{\mathrm{ex}}$ is only valid for $|\delta \lambda| \lesssim 1 / \sqrt{L}$ i.e. in the vanishingly small interval of quench amplitudes in the thermodynamic limit.

The sum of probabilities in (79) itself is not a physical observable. A measurable quantity with the same scaling is the density of created quasiparticles $n_{\mathrm{ex}}=$
$1 / L \sum_{q} n_{\mathrm{ex}}(q)$, where $n_{\mathrm{ex}}(q)=\left\langle a_{q}^{\dagger} a_{q}\right\rangle$. In the limit $p_{\text {ex }}(q) \ll 1$ the sum in (74) is dominated by the first term, $n=1$, corresponding to the creation of one pair of quasiparticles with opposite momenta. Thus in this limit $n_{\mathrm{ex}}(q) \approx 2 p_{\mathrm{ex}}(q)$. From this we conclude that for the small amplitude quenches $\lambda \lesssim \lambda_{s}$ we have $n_{\mathrm{ex}} \approx 2 p_{\mathrm{ex}}$, with $p_{\mathrm{ex}}=P_{\mathrm{ex}} / L$ given by Eq. (78). As we will see the same relation is true if we are dealing with fermionic systems. Physically this relation comes from the fact that we are dealing with few-body operators. Thus in the lowest order of perturbation theory the coupling through the quench dynamics leads to generating a small number of quasiparticles in each momentum mode. If the system is noninteracting (or more generally integrable) then the number of these quasiparticles is conserved in time after the quench. In nonintegrable systems $n_{\mathrm{ex}}$ changes in time due to scattering processes. Therefore the scaling of $n_{\text {ex }}$ with $\lambda_{f}$ is expected to be valid only either at times shorter than the relaxation time, or if the generated defects are topologically protected like in the Kibble-Zurek mechanism [32, 33]. We note that the scaling of the heat is insensitive to the relaxation processes because the energy in a closed system is conserved after the quench process. The same is true about the (diagonal) entropy.

For finite quench amplitudes $\lambda \geq \lambda_{s}$ the expressions for $n_{\mathrm{ex}}(q)$ and $1 / L \sum_{q} p_{\mathrm{ex}}(q)$ are different. In particular, it is easy to check that

$$
n_{\mathrm{ex}}(q)=\frac{\left(\sqrt{\kappa_{q}\left(\lambda_{f}\right)}-\sqrt{\kappa_{q}(0)}\right)^{2}}{4 \sqrt{\kappa_{q}\left(\lambda_{f}\right) \kappa_{q}(0)}} .
$$

For $q \gg 2 \lambda_{f} \beta^{2}$ we find that $n_{\mathrm{ex}}(q) \approx|\lambda|^{2} \beta^{4} /\left(4 q^{4}\right) \approx$ $2 p_{\mathrm{ex}}(q)$. In the opposite limit $n_{\mathrm{ex}}(q) \approx \sqrt{2 \lambda \beta^{2}} / q$ while $p_{\text {ex }}(q) \approx 1$. The difference between $n_{\text {ex }}(q)$ and $p_{\text {ex }}(q)$ happens because the states with more than two quasiparticles per each mode are excited for small momenta. Such states contribute differently into the probability of excitation and the quasiparticle density. For the total density of quasiparticles we thus find:

$$
n_{\mathrm{ex}} \approx \frac{1}{2 \pi} \int_{\frac{2 \pi}{L}}^{\Lambda} d q n_{\mathrm{ex}}(q) \approx \frac{\sqrt{2 \lambda_{f} \beta^{2}}}{4 \pi} \log \left(\frac{\lambda_{f} \beta^{2} L^{2}}{2 \pi^{2}}\right)
$$

where $\Lambda$ is the upper momentum cutoff. Note that this integral gives additional logarithmic dependence on both the quench amplitude and the system size. This dependence appears because of the infrared divergence of the density of excited quasiparticles at small $q$. It is very similar in nature to the onset of the non-adiabatic regime for slow quenches [55] and is due to the bunching effect. As we will argue later this divergence disappears in dimensions higher than one where the scalings of both $n_{\mathrm{ex}}$ and $\sum_{q} p_{\text {ex }}(q) / L^{d}$ with the quench amplitude become identical. In one dimension the two scalings agree apart from a weak logarithmic correction.

Similarly we can analyze the heat density, or equivalently the non-adiabatic energy change in the system due to the quench. For $\lambda<\lambda_{s}$ we can find, similarly to 
Eq. (78):

$$
Q=\frac{1}{L} \sum_{q>0} \sqrt{\kappa_{q}\left(\lambda_{f}\right)} n_{\mathrm{ex}}(q) \approx \frac{1}{L} \sum_{q>0} \frac{\beta^{4} \lambda_{f}^{2}}{4 q^{3}}=\frac{L^{2} \beta^{4} \lambda_{f}^{2}}{4(2 \pi)^{3}} \zeta(3) .
$$

In the opposite limit $\lambda>\lambda_{s}$ we have:

$$
\begin{aligned}
Q & \approx \frac{1}{2 \pi} \int_{\frac{2 \pi}{L}}^{\Lambda} d q \sqrt{q^{2}+2 \lambda_{f} \beta^{2}} n_{\mathrm{ex}}(q) \\
& \approx \frac{\lambda_{f} \beta^{2}}{2 \pi} \log \left(\frac{\lambda_{f} \beta^{2} L^{2}}{2 \pi^{2}}\right) .
\end{aligned}
$$

Up to the logarithmic correction in the above equation, these results agree with the prediction in Sec. II $Q \sim \lambda^{2} \chi_{E}(\lambda)$ with $\chi_{E}(\lambda) \sim L^{2 / \nu-(d+z)}$ for $\lambda<\lambda_{s}$ and $\chi_{E}(\lambda) \sim|\lambda|^{(d+z) \nu-2}$ for $\lambda>\lambda_{s}$. We point out that if we consider an opposite process where we start with a small initial coupling $\lambda_{i}$ and end at the critical point, then the expression for $n_{\mathrm{ex}}$ will remain the same, while the expression for the heat for $\lambda>\lambda_{s}$ will change because the energy is now evaluated at the critical point. This will remove the extra logarithmic divergence and we will simply get $Q \propto \lambda_{i} \beta^{2}$.

Finally let us consider the density of the (d-)entropy generated in the quench. Like $P_{\text {ex }}$ it gives the measure of the excitation of the system, however, it is explicitly extensive and has well defined scaling properties both for $\lambda<\lambda_{s}$ and $\lambda>\lambda_{s}$. Unlike $n_{\mathrm{ex}}$, the entropy (like the heat) can be defined for any system, integrable or not. Because all momentum modes are independent we find that $S_{d}=1 / L \sum_{q>0} s_{q}$, where

$$
s_{q}=-\sum_{n \geq 0}\left|c_{2 n}(q)\right|^{2} \log \left(\left|c_{2 n}(q)\right|^{2}\right) .
$$

For $\lambda \ll \lambda_{s}$ the entropy of each mode is dominated by the lowest excitation: $s_{q} \approx-\left|c_{2}(q)\right|^{2} \log \left|c_{2}(q)\right|^{2} \mid=$ $-p_{\text {ex }}(q) \log p_{\text {ex }}(q)$, therefore:

$$
S_{d} \approx-\frac{L^{3} \beta^{4} \lambda_{f}^{2}}{8(2 \pi)^{4}} \zeta(4) \log \left(\frac{\left|\lambda_{f}\right|^{2} \beta^{4} L^{4}}{8(2 \pi)^{4}}\right) .
$$

We note that for $\lambda<\lambda_{s}$ the argument of the logarithm is smaller then one so the entropy is positive as it should. In the opposite limit $\lambda>\lambda_{s}$ we find:

$$
S_{d} \approx 0.14 \sqrt{\lambda_{f} \beta^{2}} .
$$

So we see that the entropy has the same scaling as $n_{\mathrm{ex}}$ and $1 / L \sum_{q} p_{\text {ex }}(q)$.

A similar analysis can be done also in the case of a linear quench, $r=1$ and $v=\delta$. As it has been shown in Ref. [50], the scalings for the density of excited quasiparticles: $n_{\mathrm{ex}} \sim \delta^{1 / 3}$ and for the heat, $Q \sim \delta^{2 / 3}$, in the case of quenches with rate $\delta \gg 1 / L^{3}$ agree with the predictions in Eqs. (13) and (17). Similarly it is easy to check the correct scalings in the other regime, $\delta \ll 1 / L^{3}$. For instance in this regime using the expression for the number of excitation per mode $q$ (see Eq. (103) in Ref. [50]), we obtain:

$$
n_{\mathrm{ex}}=\frac{1}{L} \sum_{q} n_{q}=\frac{1}{L} \sum_{q} \frac{\left(\beta^{2} \delta\right)^{2}}{16 q^{6}}=\frac{L^{5}\left(\beta^{2} \delta\right)^{2}}{16(2 \pi)^{6}} \zeta(6),
$$

confirming the general scaling (12), which for $\nu=1 / 2$, $d=1, z=1$, and $r=1$ gives $n_{\mathrm{ex}} \sim L^{5} \delta^{2}$.

Generalization to higher dimensions. Let us note that the Hamiltonian (69) can be analyzed in any spatial dimension. The quench process simply describes the response of the free bosonic theory to a sudden turn on of the mass term. Since all the momentum modes are independent from each other the expressions for $c_{2 n}(q)$, $p_{\text {ex }}(q), n_{\text {ex }}(q)$ and $s_{q}$ remain the same. The only difference with one dimension is that we have to sum over all modes in the d-dimensional space. This introduces an additional density of states factor $\rho(q) \propto q^{d-1}$ into all the expressions. Let us focus only on quenches with $\lambda>\lambda_{s}$. It is easy to check that for $1<d<4$ one can rescale the momentum $q=\sqrt{2 \lambda_{f} \beta^{2}} \eta$ and set the limits of integration over $\eta$ to $[0, \infty)$ when analyzing $1 / L \sum_{q} p_{\text {ex }}(q)$, $n_{\mathrm{ex}}$, and $S_{d}$. This follows from the fact that at large $q$ we have $p_{\text {ex }}(q) \sim 1 / q^{4}$ so the integrals over momenta converge and the upper cutoff can be sent to infinity. Likewise in all dimensions larger than one there are no infrared divergencies in all the integrals so the lower limit of integration over $q: q_{\min } \approx 2 \pi / L$ can be sent to zero. Then for all these quantities we get the same desired scaling, e. g. $n_{\mathrm{ex}} \approx C_{d}\left(\lambda_{f} \beta^{2}\right)^{d / 2}$, where $C_{d}$ is a number which depends only on the dimensionality. Similarly for the heat for $d \leq 3$ we get $Q \approx \tilde{C}_{d}\left(\lambda_{f} \beta^{2}\right)^{(d+1) / 2}$. Above four dimensions (three dimensions for the heat) the integrals over momentum become ultraviolet divergent. So for $d>3$ the leading contribution to heat will be determined by the large momentum asymptotic of the transition probability:

$$
Q \approx 2 \frac{\lambda_{f}^{2} \beta^{4}}{8} \int \frac{d^{d} q}{(2 \pi)^{d}} \frac{q}{q^{4}} \approx C\left(\lambda_{f}^{2} \beta^{4}\right)|\Lambda|^{d-3},
$$

where $\Lambda \sim \pi$ is the high-momentum cutoff and $C$ is a non-universal constant. A similar expression is valid for $n_{\mathrm{ex}} \approx 1 / L \sum_{q} p_{\mathrm{ex}}(q)$ and for the entropy above four dimensions with the cutoff entering in the power $\Lambda^{d-4}$. For the entropy there is an additional logarithmic factor $\log \left(\lambda_{f}^{2} \beta^{4} / \Lambda^{4}\right)$. In three (four) dimensions heat (density of quasiparticles) acquire an additional logarithmic dependence on the quench amplitude: $Q \sim \lambda_{f}^{2} \beta^{4} \log \left(\Lambda /\left(\lambda_{f} \beta^{2}\right)\right)$. This divergence indicates the crossover from quadratic to universal leading asymptotics. In Sec. $\mathrm{V}]$ we already discussed how similar logarithmic corrections show up in heat in one dimensional SG model when $K=1$ for $Q\left(K=3 / 2\right.$ for $\left.n_{\mathrm{ex}}\right)$.

Using the free bosonic theory it is also easy to verify that for $3<d<5$ the first subleading asymptotic in the expansion of $Q$ in powers of $\lambda_{f}$ is non-analytic and cutoff 
independent. This follows from the fact that:

$$
\begin{aligned}
& Q=\int \frac{d^{d} q}{(2 \pi)^{d}} \sqrt{q^{2}+2 \lambda_{f} \beta^{2}}\left(n_{\mathrm{ex}}(q)-\frac{\lambda_{f}^{2} \beta^{4}}{4 q^{4}}\right) \\
& +\int \frac{d^{d} q}{(2 \pi)^{d}} q \frac{\lambda_{f}^{2} \beta^{4}}{4 q^{4}} \approx C \lambda_{f}^{2} \beta^{4}|\Lambda|^{d-3}+B\left|\lambda_{f} \beta^{2}\right|^{\frac{d+1}{2}} .
\end{aligned}
$$

Since $n_{\mathrm{ex}}(q)-\lambda_{f}^{2} \beta^{4} / 4 q^{4} \sim-\lambda_{f}^{3} \beta^{6} / 2 q^{6}$ the first integral in the above equation is ultraviolet convergent below five dimensions and we can use the rescaling $q=\sqrt{2 \lambda_{f} \beta^{2}} \eta$ and send limits of integration over $\eta$ to $[0, \infty)$. We can continue and get the asymptotical expression for the heat in high dimensions similar to Eq. (59)

$Q=\sum_{2<n<[(d+1) / 2]} C_{n}\left|\lambda_{f}\right|^{n}+B\left|\lambda_{f}\right|^{(d+1) / 2}+o\left(|\lambda|^{(d+1) / 2}\right)$.

We note that this analytic expansion goes in both even and odd powers of $\lambda_{f}$ (unlike Eq. (59) ) where only even powers of $\lambda$ appear. This has to do with the fact that for the massive bosonic theory (unlike the SG model) $\lambda \rightarrow-\lambda$ is not a symmetry of the Hamiltonian. Moreover, the theory is well defined only for $\lambda>0$. As in Eq. (59) we find logarithmic corrections when nonanalytic and analytic powers coincide, i.e. when $(d+1) / 2$ becomes an integer. A very similar expression holds for $n_{\text {ex }}$ with the only difference that in Eq. (90) one needs to replace $d+1$ with $d$. We point out that for small amplitude quenches $\lambda<\lambda_{s}$ the expansion (90) will remain valid with the first non-universal analytic terms being unaffected, while in the last non-analytic terms one needs to substitute $\left|\lambda_{f}\right|^{(d+1) / 2}$ to $\lambda_{f}^{2} L^{3-d}$. Therefore above three dimensions for quenches of small amplitude the non-analytic correction to the heat becomes sub-extensive. The same is true for the density of quasiparticles where the non-analytic term scales as $\lambda_{f}^{2} L^{4-d}$.

\section{Free massive fermions}

Another important application of the SG Hamiltonian (22) to physical systems feasible in cold atoms experiments, is given by the description of the loading process of a one-dimensional Bose gas into a commensurate optical lattice. In this case $\beta$ is related to the Luttinger parameter through: $\beta=2 \sqrt{\pi K}$, and the amplitude of the optical lattice $V(t)$ is directly proportional to $\lambda[9,13]$. This process can be studied exactly in the Tonks-Girardeau regime, i.e. when $K=1$. In this case the repulsive interaction between the bosons is infinitely strong and the particles behave as impenetrable spheres (hard-core bosons). It is well known that in this limit the system can be mapped into an equivalent system of free spinless fermions [70]. Therefore the dynamical problem of loading hard-core bosons into a commensurate optical lattice can instead be approached with the much simpler analysis of free massive fermions in a periodic po- tential. We note that this fermionic limit also describes the transverse field Ising model via the Jordan-Wigner transformation [6]. Then the quench dynamics is performed by suddenly change the transverse field starting at the critical point.

To understand the dynamics in the TG-gas limit, we need to solve the Schrödinger equation describing free fermions in a periodic commensurate potential with time dependent amplitude $V(x, t)=V(t) \cos \left(2 q_{f} x\right)$, where $q_{f}=\pi / a$ is the Fermi momentum and $a$ is the lattice spacing, which is also equal to the inverse particle density. In the TG-limit the sound velocity $v_{s}=v_{f} / K$ is equal to the Fermi velocity $v_{f}$. It is convenient to work in units such that $a=1, v_{f}=1$ and $\hbar=1$. The free fermion Hamiltonian becomes equivalent to the SG Hamiltonian (22) if we additionally restrict the analysis to the two lowest bands of the Brillouin zone and linearize the spectrum close to the Fermi momentum. Then for each momentum the loading process is described by a Landau-Zener Hamiltonian [71] (see also Ref. [31] for the discussion of the equivalent transverse field Ising model):

$$
\mathcal{H}_{k} \approx\left[\begin{array}{cc}
V(t) / 2 & k \\
k & -V(t) / 2
\end{array}\right]
$$

where we defined the momentum $k=q-q_{f}$, so that is measured from the Fermi momentum. By matching this spectrum with the one of the SG model we find that $\lambda=V /(4 \pi)$. The extra factor of $4 \pi$ appears because of the details of the bosonization procedure 72,73 .

The Hamiltonian (91) can be diagonalized on the basis of its eigenvectors: $\left|+_{k}\right\rangle$ and $\left|-{ }_{k}\right\rangle$, with respectively positive and negative energy. To study the probability of exciting the system due to a sudden quench of the coupling $V: V(t)=V_{f} \theta(t)$, we need to evaluate the overlap of the initial ground state $\left|-{ }_{k}\right\rangle_{0}$ with the final excited state $\left|+{ }_{k}\right\rangle_{V_{f}}$. Therefore $p_{\text {ex }}(k)=\left|V_{V_{f}}\left\langle+_{k} \mid-{ }_{k}\right\rangle_{0}\right|^{2}$. In particular we find:

$$
p_{\mathrm{ex}}(k)=\frac{1}{2} \frac{V_{f}^{2}+k^{2}-V_{f}|k|+\left(V_{f}-|k|\right) \sqrt{V_{f}^{2}+k^{2}}}{V_{f}^{2}+k^{2}+V_{f} \sqrt{V_{f}^{2}+k^{2}}} .
$$

This transition probability has the following asymptotics: $p_{\mathrm{ex}}(k) \approx V_{f}^{2} /\left(4 k^{2}\right)$ for $k \gg V_{f}$ and $p_{\mathrm{ex}}(k) \approx 1 / 2$ for $k \ll V_{f}$. As in the previous case of free bosons, the elementary excitation correspond to the creation of particlehole pairs moving with opposite momenta (recall that annihilating a particle in the lower band can be viewed as creating a hole there with an opposite momentum). However, unlike with bosons, we can not create more than one pair for each momentum state due to the Pauli exclusion principle. This means that $1 / L \sum_{k} p_{\text {ex }}(k)$ and $n_{\text {ex }}$ are always identical up to a factor of two. As in the bosonic case the crossover scale $V_{s}$ can be defined from the requirement that $\sum_{k} p_{\mathrm{ex}}(k) \ll 1$, which gives $V_{s} \sim 4 \pi / L$ (or equivalently $\lambda_{s} \sim 1 / L$ ). For $V<V_{s}$ the total probability to excite the system is small so that $P_{\mathrm{ex}} \simeq \sum_{k} p_{\mathrm{ex}}(k)$. This characteristic scale agrees with 
the expected one $\lambda_{s} \simeq L^{-1 / \nu}$ from the analysis of the system close to the quantum critical point, since in this case $\nu=1$. Using the explicit asymptotics for the excitation probability $p_{\text {ex }}(k)$ at small amplitudes $V_{f}$ we find that in this case:

$$
\frac{P_{\mathrm{ex}}}{L} \approx \frac{n_{\mathrm{ex}}}{2} \approx \frac{V_{f}^{2} L}{48}=\frac{\pi^{2}}{3} \lambda_{f}^{2} L .
$$

For large quenches $V_{f} \gg V_{s}$ we find that:

$$
n_{\mathrm{ex}} \approx 4 \int_{-\pi}^{0} \frac{d k}{2 \pi} p_{\mathrm{ex}}(k)=\frac{V_{f}}{\pi}=4 \lambda_{f} .
$$

We see that as in the case of bosons $n_{\text {ex }}$ has the correct scaling $n_{\mathrm{ex}} \sim\left|\lambda_{f}\right|^{d \nu}$ for $\lambda>\lambda_{s}$ and $n_{\mathrm{ex}} \sim \lambda_{f}^{2} L^{2 / \nu-d}$ for $\lambda<\lambda_{s}$ in agreement with Eqs. (12) and (13) respectively.

Likewise one can evaluate the heat density $Q=$ $2 / L \sum_{k} p_{\mathrm{ex}}(k) \sqrt{k^{2}+V_{f}^{2} / 4}$. For $\lambda<\lambda_{s}$ we find:

$$
\begin{aligned}
Q & =\frac{2}{L} \sum_{k} \sqrt{k^{2}+V_{f}^{2} / 4} p_{\mathrm{ex}}(k) \\
& \approx \frac{V_{f}^{2}}{2 \pi} \log (L / 2)=8 \pi \lambda_{f}^{2} \log (L / 2),
\end{aligned}
$$

and in the opposite limit $\lambda>\lambda_{s}$ :

$$
Q=\frac{V_{f}^{2}}{8 \pi} \log \left(2 \pi / V_{f}\right)=-2 \pi \lambda_{f}^{2} \log \left(2 \lambda_{f}\right) .
$$

This scaling of heat agrees with the general expectations of Eqs. (16) and (17) and the results obtained in the previous sections using the adiabatic perturbation theory. Since we have $(d+z) \nu=2$, there is an additional logarithmic correction indicating the crossover from quadratic to nonanalytic scaling in the leading asymptotic of the scaling of $Q$ with $\lambda_{f}$. Note that the difference in scaling between $\lambda<\lambda_{s}$ and $\lambda>\lambda_{s}$ only appears in the logarithmic term.

Finally we can evaluate the entropy density. Let us explicitly quote only the result for $\lambda>\lambda_{s}$ :

$$
\begin{aligned}
S_{d} & =-\frac{1}{L} \sum_{k}\left[\left[p_{\text {ex }}(k) \log \left(p_{\text {ex }}(k)\right)\right.\right. \\
& +\left(1-p_{\text {ex }}(k) \log \left(1-p_{\text {ex }}(k)\right)\right]=\frac{V_{f}}{2}=2 \pi \lambda_{f} .
\end{aligned}
$$

The scaling of the entropy is thus again in accord with the exact solution. It is easy to check that for $\lambda<\lambda_{s}$ the entropy density scales as $S \sim \lambda_{f}^{2} L \log \left(\lambda_{f} L\right)$, i.e. it is super-extensive.

As in the bosonic case, these predictions can be also checked in the case of slow quenches. In Ref. [50] it has been shown that for the linear quench $\lambda(t)=\delta t$ one finds $n_{\mathrm{ex}} \sim \sqrt{\delta}$ for $\delta \gg 1 / L^{2}$, in agreement with Eq. (13), while for $\delta \ll 1 / L^{2}$ one can find that (see Eq. (108) and Appendix $\mathrm{A}$ in [50] for the expression for $n_{q}$ ):

$$
n_{\mathrm{ex}}=\frac{1}{L} \sum_{k} n_{k}=\frac{1}{L} \sum_{k} \frac{4 \delta^{2}}{k^{4}}=\frac{4 L^{3} \delta^{2}}{(2 \pi)^{4}} \zeta(4),
$$

which confirms the scaling $n_{\mathrm{ex}} \sim L^{3} \delta^{2}$ given by Eq. (12).

Generalization to higher dimensions. Like in the case of bosons one can extend the analysis to higher dimensional free fermionic models. For example, the twodimensional generalization of the free fermionic theory describes commensurate fermions in the honeycomb lattice, which are realized in graphene [74]. Such lattices can be also in principle realized in cold atoms using multiple laser beams 75 . The quench process corresponds to the distortion of the lattice, which opens a gap in the spectrum. Quench dynamics in similar setup was recently analyzed in Ref. [43, 76].

Unlike in the bosonic limit, where $\nu=1 / 2$, here we have $\nu=1$. This implies that we expect that the quadratic scaling for the quasiparticle density and entropy emerges above two dimensions and for the heat above one dimension. This is indeed the case since the excitation probability at large momenta scales as $p_{\text {ex }}(k) \sim 1 / k^{2}$ (versus $1 / k^{4}$ in the case of bosons). To understand the general structure of the heat density above one dimension we can more closely examine the expansion of the transition probability $p_{\text {ex }}(k)$ at large $k$. In particular, from Eq. (92) we find:

$$
p_{\mathrm{ex}}(k) \approx \frac{V_{f}^{2}}{4 k^{2}}-\frac{3 V_{f}^{4}}{16 k^{4}}+\frac{5 V_{f}^{6}}{32 k^{6}}+\ldots
$$

As we see the expansion is in even powers of $V_{f}$ which reflects the symmetry of the problem with respect to $V_{f} \rightarrow-V_{f}$. For $1<d<3$ we can rewrite the heat as

$$
\begin{aligned}
Q & \approx 2 M \int_{-\pi}^{0} \frac{d^{d} k}{(2 \pi)^{d}} \sqrt{k^{2}+V_{f}^{2} / 4}\left(p_{\mathrm{ex}}(k)-\frac{V_{f}^{2}}{4 k^{2}}\right) \\
& +2 M \int_{-\pi}^{0} \frac{d^{d} k}{(2 \pi)^{d}} \frac{V_{f}^{2}}{4 k},
\end{aligned}
$$

where $M$ is the number of independent light cones in the system ( $M=2$ for graphene). The second contribution gives a non-universal quadratic term, while in the first integral we can rescale the variables $k \rightarrow V_{f} \eta$ and send the limits of integration over $\eta$ to $[0, \infty)$. This contribution thus gives the non-analytic term $\left|V_{f}\right|^{(d+z) \nu}$. A similar analysis can be extended to higher dimensions and as a result we get the following expansion:

$$
Q=\sum_{1<n<[(d+1) / 2]} C_{n}\left|\lambda_{f}\right|^{2 n}+B\left|\lambda_{f}\right|^{d+1}+o\left(|\lambda|^{d+1}\right) .
$$

The coefficients $C_{n}$ are non-universal (cutoff dependent) while the coefficient $B$ is universal and can be obtained from the low energy description of the system. Similar considerations can be applied to the density of quasiparticles resulting in the expression similar to Eq. (101) with $d+1 \rightarrow d$. For small amplitude quenches $\lambda<\lambda_{s}$ only the universal term in the expansion above gets modified with $\left|\lambda_{f}\right|^{d+1} \rightarrow \lambda_{f}^{2} L^{1-d}$. As we already mentioned in the points where the power of the non-analytic term crosses the one of the analytic contribution $(d+1=2 n)$ there are additional logarithmic corrections to the heat. 


\section{Finite temperature quenches}

Let us extend the analysis of these thermodynamic quantities to the case of a finite initial temperatures. We suppose to perform the same quench process but now preparing the system initially in a thermal state at temperature $T$. We will assume that the system is isolated during the dynamical process. For instantaneous quenches this assumption is generally always satisfied. For slow quenches this assumption is only valid if we are interested in the time scales shorter than the relaxation time with the thermal bath. This is the usual requirement in order to be in the adiabatic limit in conventional thermodynamics [77] and it is usually well satisfied in cold atoms. We note that other studies have addressed the case of systems where the contact with a thermal bath during the dynamical process is essential 78 . The advantage of focusing on the setup where the temperature enters only through the initial conditions and does not affect the equations of motion is that one can expect that the scaling of various quantities will remain universal. We note that finite temperature dynamics in this setup were studied earlier in Refs. [50, 55] for slow linear quenches and in Ref. [19] for sudden quenches.

\section{Free massive bosons.}

For the case in consideration of a system of independent harmonic oscillators (see Eq. (69)), a finite initial temperature leads to the occupation of the eigen-modes according to the Bose-Einstein distribution:

$$
n_{T}(q)=\frac{1}{\exp \left[\beta \sqrt{\kappa_{q}(0)}\right]-1}=\frac{1}{\exp [\beta q]-1} .
$$

This initial occupation enhances the transition probabilities. In Ref. [55] it was shown that for an arbitrary time dependence of $\lambda(t)$, the mode occupation with momentum $q$ at finite temperature is related to the one at zero temperature via:

$$
\begin{aligned}
n_{\mathrm{ex}}^{\mathrm{tot}}(q) & =n_{\mathrm{ex}}(q) \operatorname{coth}\left[\frac{\sqrt{\kappa_{q}(0)}}{2 T}\right]+n_{T}(q) \\
& =n_{\mathrm{ex}}(q) \operatorname{coth}\left[\frac{|q|}{2 T}\right]+n_{T}(q) .
\end{aligned}
$$

where $n_{\mathrm{ex}}^{\text {tot }}(q)$ is the total number of excited quasiparticles during the dynamical process, that is indeed equal to the initial thermal population of this mode $\left(n_{T}(q)\right)$, plus the number of particles generated in the identical process at zero temperature multiplied by a hyperbolic cotangent factor. Therefore we can define the excitations $n_{\mathrm{ex}}^{T}(q)$ created only by the dynamical process at a finite temperature as the difference $n_{\mathrm{ex}}^{\text {tot }}(q)-n_{T}(q)$. It is immediately clear that the hyperbolic factor gives an enhancement of the transitions since at $T \gg q$ it scales as $2 T / q \gg 1$. The result (103) can be seen either from the thermal average of the energy of each mode $q$ as it was recently pointed out in Ref. [19], or writing the initial thermal state in the Wigner form, as shown in Ref. [55].

Let us now analyze how this additional factor affects the scaling of the quantities of interest. To simplify the discussion we will focus only on the heat density. We will also assume that the temperature is large compared to both the energy associated with the quench amplitude $T \gg \sqrt{\lambda_{f} \beta^{2}}$ and the finite size quantization $T \gg 1 / L$. Otherwise we will return to the zero temperature asymptotics.

For $\lambda<\lambda_{s}$ by simple modification of Eq. (82) we find

$$
Q \approx \frac{2 T}{L} \sum_{q>0} \frac{\beta^{4} \lambda_{f}^{2}}{4 q^{4}}=\frac{T L^{3} \beta^{4} \lambda_{f}^{2}}{2(2 \pi)^{4}} \zeta(4) .
$$

In the opposite limit $\lambda>\lambda_{s}$ instead of Eq. (83) we find:

$$
Q \approx \frac{2 T}{L} \sum_{q>0} \frac{2 \lambda_{f} \beta^{2}}{q^{2}} \approx \frac{1}{6} T L \lambda_{f} \beta^{2} .
$$

For small quenches the result (104) is fully consistent with the scaling prediction of the Eq. (16): $Q \sim$ $\lambda_{f}^{2} L^{2 / \nu-d}$. While for larger quench amplitudes we get a deviation. Instead of $Q \sim\left|\lambda_{f}\right|^{d \nu}=\sqrt{\left|\lambda_{f}\right|}$ we get the scaling $Q \sim\left|\lambda_{f}\right| L$. The origin of this discrepancy is the infrared divergence of the sum in Eq. (105) at small momenta. This divergence is similar to the one appearing in linear quenches, which leads to the non-adiabatic regime [55]. As it is easy to check this divergence disappears in higher dimensions and the predicted general scaling is restored. Indeed for $2<d<4$ and for $\lambda>\lambda_{s}$ one can substitute the summation over momenta in the general expression for $q$ by an integration, change the variables $q=\sqrt{2 \lambda_{f} \beta^{2}} \eta$ and extend the limits of integration over $\eta$ to $[0, \infty)$. This immediately implies that $Q \sim T\left|\lambda_{f} \beta^{2}\right|^{d / 2}$. Above four dimensions the integral over momenta becomes ultra-violet divergent. In this case the transitions are dominated by high momenta for which the initial thermal occupation is not important (if the temperature is much smaller than the high-energy cutoff: $T \ll \pi$ in our units) and we are back to the zero temperature non-universal result (88). It is easy to check that in this case (as at zero temperatures) the universal nonanalytic correction to $Q$ becomes subleading thus instead of Eq. (90) we find:

$$
Q=\sum_{2<n<[d / 2]} C_{n}\left|\lambda_{f}\right|^{n}+B T\left|\lambda_{f}\right|^{d / 2}+o\left(|\lambda|^{d / 2}\right) .
$$

To summarize the finite temperature results for the bosonic systems we see that the substitution $d \rightarrow d-z$ always predicts the correct finite temperature scaling of the heat for $\lambda<\lambda_{s}$. In the opposite limit $\lambda>\lambda_{s}$ this substitution $d \rightarrow d-z$ also works in two dimensions and above but fails in one dimension. The same applies to the density of generated quasiparticles. 


\section{Free massive fermions.}

It is also straightforward to obtain the finite temperature asymptotics in the fermionic case. Since in this case we are dealing with a sum of independent two-level systems, at finite temperature each upper $\left|+{ }_{k}\right\rangle$ and lower $\left|-_{k}\right\rangle$ level is occupied according to the Fermi distribution:

$$
f_{k}^{ \pm}=\left(\exp \left[ \pm \frac{|k|}{T}\right]+1\right)^{-1}
$$

where we used the convention that the Fermi velocity is one so that the energy of the two states $\epsilon_{ \pm}(k) \approx \pm k$. It is well known that for the two-level system the transition probability satisfies the detailed balance, i.e. the transition probability from the top to the bottom level is the same as for the opposite process (for systems with more than two levels the detailed balance is generally not true, see e.g. Ref. [47]). Therefore the number of additionally excited particles in the dynamical process gets corrected as:

$$
n_{\mathrm{ex}}^{T}(k)=n_{\mathrm{ex}}(k)\left(f_{k}^{-}-f_{k}^{+}\right)=n_{\mathrm{ex}}(k) \tanh \left(\frac{|k|}{2 T}\right) .
$$

This expression is similar to the one for the bosonic case (103), with now the hyperbolic tangent factor replacing the cotangent one. Contrary to the bosonic case therefore, the effect of the finite temperatures is to suppress the transitions since $|\tanh (x)|<1$. At high temperatures $T \gg k$ the suppression factor is approximately $|k| /(2 T)$.

As in the case of bosons let us focus on the scaling of heat. Note that the main contribution to Eqs. (95) and (96) even at zero temperature comes from high energies $k \sim \Lambda$. Therefore in the leading order in $\lambda_{f}$ the temperature will not significantly modify the expression for the heat except changing the cutoff in the logarithm:

$$
Q \approx 8 \pi \lambda_{f}^{2} \log (\pi / T) .
$$

This scaling is valid both for $\lambda<\lambda_{s}$ and $\lambda>\lambda_{s}$. This is indeed an anticipated result because the universal power $(d+2 z) \nu=3$ is bigger than two. The next subleading correction in $\lambda_{f}$ remains universal though and scales as $\lambda_{f}^{2} / L$ for $\lambda<\lambda_{s}$ and $\left|\lambda_{f}\right|^{3} / T$ for $\lambda>\lambda_{s}$. It is straightforward to check that this general structure of the expansion extends to higher dimensions so that for $\lambda>\lambda_{s}$ instead of Eq. (101) we find:

$$
Q=\sum_{1<n<[(d+2) / 2]} C_{n}\left|\lambda_{f}\right|^{2 n}+\tilde{B}\left|\lambda_{f}\right|^{d+2}+o\left(|\lambda|^{d+2}\right) .
$$

The non-universal coefficients $C_{n}$ are temperature independent (for $T \ll \Lambda$ ) while the universal coefficient $\tilde{B}$ is different from the zero-temperature one. We thus see that the finite temperature effects result in changing $d \rightarrow d+z$ in the universal part of the expression for heat. It is easy to check that the same is true for the density of excited quasiparticles.

\section{QUANTUM GEOMETRIC TENSORS AND FIDELITY SUSCEPTIBILITY. MAZUR INEQUALITIES.}

\section{A. Quantum geometric tensors: generalizations}

In the discussion above we only considered situations where $\lambda$ was a single component coupling. In principle one can extend the analysis to more general setups where $\lambda$ is a $M$-component vector in the parametric space. Within the adiabatic perturbation theory it is easy to generalize the expression for the transition probability to the many-body state $|n\rangle$ due to a sudden quench in the multicomponent case:

$$
\left|\alpha_{n}(\lambda)\right|^{2} \approx\left|\int d \boldsymbol{\lambda}^{\prime} \frac{\left\langle n\left|\partial_{\boldsymbol{\lambda}^{\prime}} H\right| 0\right\rangle}{E_{n}\left(\boldsymbol{\lambda}^{\prime}\right)-E_{0}\left(\boldsymbol{\lambda}^{\prime}\right)}\right|^{2},
$$

where the contour integral is taken over an arbitrary path connecting the initial and final couplings. For sudden quenches the precise path is clearly unimportant. Next we will use the Cauchy-Schwarz inequality, which for arbitrary square integrable functions $f(x), g(x)$ states that

$$
\left|\int f(x) g(x) d x\right|^{2} \leq \int|f(x)|^{2} d x \cdot \int|g(x)|^{2} d x .
$$

Choosing $g(x)=1$ and $f(x)$ to be the integrand in Eq. (110) we find:

$$
P_{\mathrm{ex}}=\sum_{n \neq 0}\left|\alpha_{n}(\lambda)\right|^{2} \leq M \sum_{\alpha=1}^{M} \lambda_{\alpha} \int_{0}^{\lambda_{\alpha}} d \lambda_{\alpha}^{\prime} Q_{\alpha, \alpha}\left(\lambda^{\prime}\right),
$$

where $Q_{\alpha, \alpha}(\lambda)$ are the diagonal components of the quantum geometric tensor $Q_{\alpha, \beta}(\lambda)$ defined in Ref. [53]. This tensor and its rescaled counterpart $\left(q_{\alpha \beta}=L^{-d} Q_{\alpha \beta}\right)$ has definite scaling properties close to the quantum critical point:

$$
q_{\alpha \beta}(\lambda) \sim|\lambda|^{\nu \Delta_{Q_{\alpha, \beta}}}
$$

where, according to Ref. [53]:

$$
\Delta_{\alpha \beta}^{Q}:=\Delta_{\alpha}+\Delta_{\beta}-2 z-d .
$$

We remind that $\nu$ is the correlation length critical exponent, which can in principle depend on the direction of the quench in the $\lambda$ space, and $z$ is the dynamical critical exponent. For a single-component, $\Delta_{\alpha}$ is the scaling dimension of the operator $V=\partial_{\lambda} H$. Assuming that $V$ is non-zero in the limit $\lambda \rightarrow 0$ from the scale invariance of the action we find that $\Delta_{\alpha}=d+z-1 / \nu$, which using Eq. (114) reproduces the correct scaling of the probability to excite the system $P_{\text {ex }} \sim|\lambda|^{2} L^{2 / \nu}$. Note that the diagonal components of the geometric tensor give the fidelity susceptibilities $\chi_{f}$ discussed earlier (see also Ref. 79] for the review). These observations suggest a close relation between the scalings of $P_{\text {ex }}$ with the quench amplitude, 
the quantum geometric tensors and the fidelity susceptibility characterizing the static ground state properties of the system. A similar analysis shows the connection between the heat and the energy susceptibility $\chi_{E}$ (see Eq. (49)).

The importance of the geometric tensor becomes clear in the contest of multi-parametric dynamics, when several parameters involved in the model can depend on time. In this case the integrals in the expressions for the physical quantities should be replaced by the line integrals in the parameter space. One can define a "response" in terms of one parameter by driving another parameter. The corresponding susceptibility of this response is described by the real parts of the non-diagonal matrix elements of the quantum geometric tensor. Moreover the imaginary part of the geometric tensor is responsible for the Berry phase which may appear as a result of adiabatic evolution in this multi-parameter space. We are going to come back to these questions in the future.

\section{B. Integrable perturbations and ergodicity}

It is interesting to note that the Cauchy-Schwarz inequality, although it gives simply an upper bound, it appears to be exact for the scaling functions we derived here. On the other hand for some type of perturbations away from the QCP's (the so-called integrable perturbations) it seems possible to formulate a lower bound as well. It is known [80] that for the models which have integrals of motion, the time averages of the correlation functions have a lower bound and therefore their dynamics can be non-ergodic. In particular, if the quantities $A_{i}(i=1, \cdots m)$ are the integrals of motion, $\left[H, A_{i}\right]=0$ $\forall i$, defined such that $\left\langle A_{i}\right\rangle=0$, then the dynamical correlation function of the operator $X(t)$ is bounded from below as:

$$
\lim _{T \rightarrow \infty} \frac{1}{T} \int_{0}^{T}\langle X(t) X(0)\rangle d t \geq\langle X \mathbf{A}\rangle \cdot\langle\mathbf{A} \mathbf{A}\rangle^{-1} \cdot\langle X \mathbf{A}\rangle
$$

where $\langle\mathbf{A A}\rangle$ is the $m \times m$ matrix with elements $\left\langle A_{i} A_{j}\right\rangle$. Provided that this matrix can be diagonalized by some unitary transformation and that the Hamiltonian is identified with one of the operators $A_{i}$, say $H \equiv A_{1}$, we obtain $\left\langle\tilde{A}_{i} \tilde{A}_{j}\right\rangle /\left\langle\tilde{A}_{i}^{2}\right\rangle=\delta_{i j}$, where the notation $\tilde{A}$ stands for the transformed operator, and therefore [80]

$$
\lim _{T \rightarrow \infty} \frac{1}{T} \int_{0}^{T}\langle X(t) X(0)\rangle d t \geq \sum_{k=1}^{m} \frac{\left\langle X \tilde{A}_{k}\right\rangle^{2}}{\left\langle\tilde{A}_{k}^{2}\right\rangle} \geq \frac{\langle X H\rangle^{2}}{\left\langle H^{2}\right\rangle}
$$

We implicitly assume that our Hamiltonian is defined in such a way that $\langle H\rangle=0$.

The sine-Gordon model represents an integrable deformation (perturbation) of the conformal field theory. Therefore it has many (infinite number) integrals of motion. Taking $X(t) \equiv \int_{0}^{L} \cos (\beta \phi(x, t))$ we obtain a lower bound for the time average of the energy susceptibility

$$
\chi_{E}^{(t)}=\lim _{T \rightarrow \infty} \frac{1}{T} \int_{0}^{T} \int_{0}^{L} d x G(x, \tau) d \tau .
$$

By the Mazur inequality (116) this susceptibility has a lower bound, which is nonzero. I.e. $\chi_{E}$ can either be finite or diverge. The Mazur inequalities above can be also directly generalized for other time averages of the susceptibilities like $\chi_{f}^{(t)}$ defined in Eq. (53) as well as higher order adiabatic susceptibilities [84].

\section{CONCLUSIONS}

In this work we analyzed the quench dynamics starting (ending) at the quantum critical point focusing on the sine-Gordon model. We derived the universal scalings of such quantities as the probability of exciting the system $\left(P_{\text {ex }}\right)$, density of excited quasiparticles $\left(n_{\mathrm{ex}}\right)$, entropy and heat densities $\left(S_{d}\right.$ and $\left.Q\right)$ with the quench amplitude. These scalings are fully determined by the critical exponents $z$ and $\nu$ characterizing the quantum critical point and agree with general expectation presented in [45].

In particular for the type of quenches where the tuning parameter changes as a power law near the quantum critical point: $\lambda(t) \approx v t^{r} / r$ !, we showed that the scalings of $P_{\mathrm{ex}}, n_{\mathrm{ex}}$, and $S_{d}$ are associated with the singularities of generalized adiabatic susceptibilities $\chi_{2 r+2}(\lambda)$ of order $2 r+2$ (see Eq. (7)), while if the quench ends at the critical point the scaling of $Q$ is associated with the singularity of $\chi_{2 r+1}$. We note that for $r>0$ it is sufficient to have gapless systems, not necessarily quantum critical point, in order to observe these singularities.

For the two limits where the elementary excitations in the system are characterized by free bosons or fermions (for the sine-Gordon model these limits correspond to a particular choice of $\beta$, see Eq. (22) ) we generalized our results for the finite temperature quenches. We showed that the structure of the singularities remains the same except that in the scaling relations for the density of quasiparticles and heat one needs to substitute the dimensionality $d \rightarrow d-z$ for bosons (simultaneously multiplying $Q, n_{\mathrm{ex}}$ by the temperature) and for $d \rightarrow d+z$ for fermions (simultaneously dividing $Q, n_{\mathrm{ex}}$ by the temperature). This changes are a direct manifestation of the statistics of the quasiparticles: bunching of bosons enhancing non-adiabatic effects and antibunching of fermions suppressing the transitions.

We believe our results can be directly tested both numerically and experimentally, especially in setups realizable with cold atoms.

\section{Acknowledgements}

We acknowledge discussion with D. Baeriswyl, R. Barankov and B. Gut. We also thank R. Barankov 
for pointing out the correct normalization [3] for the rapidities variables in Eq. (36). The work was supported by AFOSR YIP, NSF: DMR 0907039, and Sloan Foun- dation. C. D. G. acknowledges the support of I2CAM: DMR-0645461. V.G. was supported by the Swiss National Science Foundation.
[1] S. P. Novikov, S. V. Manakov, L. B. Pitaevskii, and V. E. Zakharov, Theory of Solitons. The Inverse Scattering Method (Plenum Press, New York, 1984).

[2] A. Zamolodchikov, Int. J. Mod. Phys. A 10, 1125 (1995).

[3] G. Mussardo, Statistical Field Theory: An Introduction to Exactly Solved Models in Statistical Physics, Oxford UP, 2009.

[4] P. M. Chaikin and T. C. Lubensky, Principles of condensed matter physics (Cambridge Univesrity Press, Cambridge, 1995).

[5] T. Giamarchi, Quantum Physics in One Dimension (Clarendon Press, Oxford, 2004).

[6] S. Sachdev, Quantum Phase Transitions (Cambridge University Press, 1999).

[7] A. Iucci and M. A. Cazalilla, Phys. Rev. A 80, 063619 (2009).

[8] V. Gritsev, E. Demler, M. Lukin, and A. Polkovnikov, Phys. Rev. Lett. 99, 200404 (2007).

[9] H. P. Büchler, G. Blatter, and W. Zwerger, Phys. Rev. Lett. 90, 130401 (2003).

[10] T. Giamarchi and H. J. Schulz, Phys. Rev. B 35, 325 (1987).

[11] A. F. Ho, M. A. Cazalilla, and T. Giamarchi, Phys. Rev. Lett. 92, 130405 (2004).

[12] F. D. M. Haldane, Journal of Physics C: Solid State Physics 14, 2585 (1981).

[13] C. De Grandi, R. A. Barankov, and A. Polkovnikov, Phys. Rev. Lett. 101, 230402 (2008).

[14] Julia S. Meyer, K. A. Matveev, J. Phys.: Condens. Matter 21, 023203 (2009).

[15] G. Refael, E. Demler, Y. Oreg, and D. S. Fisher, Phys. Rev. B 68, 214515 (2003).

[16] I. Bloch, J. Dalibard, and W. Zwerger, Reviews of Modern Physics 80, 885 (2008).

[17] M. Greiner et. al., Nature (London) 419, 51 (2002); T. Kinoshita, T. Wenger, and D. S. Weiss, ibid. 440, 900 (2006); L. E. Sadler et. al., ibid. 443, 312 (2006); S. Hofferberth et. al., ibid. 449, 324 (2007).

[18] P. Calabrese, J. Cardy, Phys. Rev. Lett. 96, 136801 (2006); J. Stat. Mech. (2007) P06008.

[19] S. Sotiriadis, P. Calabrese, J. Cardy: EPL 87 (2009) 20002.

[20] M. A. Cazalilla, Phys. Rev. Lett. 97, 156403 (2006).

[21] K. Sengupta, S. Powell, and S. Sachdev, Phys. Rev. A 69, 053616 (2004).

[22] C. Kollath, A. M. Läuchli, and E. Altman, Phys. Rev. Lett. 98, 180601 (2007).

[23] G. Roux, Phys. Rev. A 79, 021608 (2009); arXiv:0909.4620.

[24] A. Faribault, P. Calabrese, and J.-S. Caux, J. Math. Phys. 50, 095212 (2009).

[25] M. Rigol, V. Dunjko, and M. Olshanii, Nature 452, 854 (2008).

[26] M. Rigol, Phys. Rev. Lett. 103, 100403 (2009).

[27] M. Rigol, V. Dunjko, V. Yurovsky, and M. Olshanii, Phys. Rev. Lett. 98, 050405 (2007).
[28] G. Biroli, C. Kollath, A. Laeuchli: arXiv:0907.3731.

[29] A. Polkovnikov, Phys. Rev. B 72, R161201 (2005).

[30] W. H. Zurek, U. Dorner, and P. Zoller, Phys. Rev. Lett. 95, 105701 (2005).

[31] J. Dziarmaga, Phys. Rev. Lett. 95, 245701 (2005).

[32] T. W. B. Kibble, J. Phys. A 9, 1387 (1976).

[33] W. H. Zurek, Phys. Rep. 276, 177 (1996).

[34] R. W. Cherng and L. S. Levitov, Phys. Rev. A 73, 043614 (2006).

[35] V. Mukherjee, U. Divakaran, A. Dutta, and D. Sen, Phys. Rev. B 76, 174303 (2007).

[36] K. Sengupta, D. Sen, and S. Mondal, Phys. Rev. Lett. 100, 077204 (2008).

[37] T. Caneva, R. Fazio, and G. E. Santoro, Phys. Rev. B 76, 144427 (2007).

[38] U. Divakaran, A. Dutta, and D. Sen, Phys. Rev. B 78, 144301 (2008).

[39] S. Deng, G. Ortiz, L. Viola, EPL 84, (2008) 67008.

[40] D. Sen, K. Sengupta, and S. Mondal, Phys. Rev. Lett. 101, 016806 (2008).

[41] S. Mondal, K. Sengupta, and D. Sen, Phys. Rev.B 79, 045128 (2009).

[42] U. Divakaran, V. Mukherjee, A. Dutta, D. Sen, J. Stat. Mech. (2009) P02007.

[43] B. Dóra, R. Moessner: arXiv:0909.2528.

[44] S. Suzuki, D. Rossini, G. E. Santoro: arXiv:0910.4055.

[45] C. De Grandi, V. Gritsev, and A. Polkovnikov, Phys. Rev. B 81, 012303 (2010).

[46] A. Polkovnikov: arXiv:0806.2862.

[47] A. Polkovnikov, Phys. Rev. Lett. 101, 220402 (2008).

[48] D. Sen, K. Sengupta, and S. Mondal, Phys. Rev. Lett. 101, 016806 (2008).

[49] R. Barankov and A. Polkovnikov, Phys. Rev. Lett. 101, 076801 (2008).

[50] C. De Grandi, A. Polkovnikov: arXiv:0910.2236, contribution to "Quantum Quenching, Annealing and Computation", Eds. A. Das, A. Chandra and B. K. Chakrabarti, Lect. Notes in Phys., vol. 802 (Springer, Heidelberg 2010).

[51] G. Rigolin, G. Ortiz, and V. H. Ponce, Phys. Rev. A 78, 052508 (2008).

[52] W.-L. You, Y.-W. Li, S.-J. Gu, Phys. Rev. E 76, 022101 (2007); S.-J. Gu, arXiv:0811.3127; S.-J. Gu, H.-Q. Lin, EPL 87, 10003 (2009).

[53] L. C. Venuti and P. Zanardi, Phys. Rev. Lett. 99, 095701 (2007).

[54] D. Schwandt, F. Alet, and S. Capponi, Phys. Rev. Lett. 103, 170501 (2009).

[55] A. Polkovnikov and V. Gritsev, Nature Phys. 4, 477 (2008).

[56] M. Eckstein and M. Kollar, arXiv:0911.1282.

[57] V. Gritsev and A. Polkovnikov, in Developments in Quantum Phase Transitions, edited by L. Carr (to be published).

[58] F. H. L. Essler, R. M. Konik, Applications of Massive Integrable Quantum Field Theories to Problems in Con- 
densed Matter Physics, I. Kogan Memorial Volume by World Scientific, cond-mat/0412421.

[59] F. A. Smirnov, Form Factors in Completely Integrable Models of Quantum Field Theory, World Scientific, 1992.

[60] S. Lukyanov, Mod.Phys.Lett. A 12, 2543 (1997), hepth/9703190.

[61] S. Lukyanov, Commun. Math. Phys. 167, 183 (1995).

[62] R. M. Konik, A. LeClair, Nucl. Phys. B 479, 619 (1996).

[63] V. Gritsev, A. Polkovnikov, and E. Demler, Phys. Rev. B 75, 174511 (2007)

[64] A. B. Zamolodchikov and A. B. Zamolodchikov, Annals of Physics 120, 253 (1979).

[65] H. Babujian, M. Karowski, Int. J. Mod. Phys. A 19S2, 34 (2004).

[66] D. Controzzi, F. H. L. Essler, A. M. Tsvelik, condmat/0011439.

[67] A. B. Zamolodchikov, Int. J. Mod. Phys. A4 (1989) 4235.

[68] C. Destri, H. J. de Vega, Nucl.Phys. B438, 413 (1995).

[69] G. Feverati, F. Ravanini, G. Takacs, Nucl.Phys. B540, 543 (1999); G. Feverati, hep-th/0001172.

[70] M. Girardeau, J. Math .Phys. 1, 516 (1960); C. N. Yang and Y. P. Yang, J. Math .Phys. 10, 1115 (1969); L. Tonks, Phys. Rev. 50, 955 (1936).

[71] L. D. Landau, Phys. Z. Sowjetunion 2, 46 (1932); C. Zener, Proc. R. Soc. London, Ser. A 137, 696 (1932).

[72] C. Mora and Y. Castin, Phys. Rev. A 67, 053615 (2003).

[73] M. A. Cazalilla, J. Phys. B 37, S1 (2004).

[74] A. H. C. Neto, F. Guinea, N. M. R. Peres, and K. S. N. andA. K. Geim, Rev. Mod. Phys. 81, 109 (2009).
[75] G. Grynberg, B. Lounis, P. Verkerk, J.-Y. Courtois, and C. Salomon, Phys. Rev. Lett. 70, 2249 (1993).

[76] A. Dutta, R. R. P. Singh, and U. Divakaran, arXiv:0910.3896 (unpublished).

[77] L. D. Landau and E. M. Lifshitz, Statistical Physics, Third Edition, Part 1: Volume 5 (ButterworthHeinemann, 1980).

[78] D. Patanè, A. Silva, L. Amico, R. Fazio, and G. E. Santoro, Phys. Rev. Lett. 101, 175701 (2008); D. Patanè, A. Silva, F. Sols, L. Amico, Phys. Rev. Lett. 102, 245701 (2009); D. Patanè, L. Amico, A. Silva, R. Fazio, and G. E. Santoro, Phys. Rev. B 80, 024302 (2009).

[79] S.-J. Gu, arXiv:0807.3491.

[80] P. Mazur, Physica, 43 (1969) 533-545.

[81] By doing this we avoid the difficulties related to the differences in notations which are common in the literature.

[82] One can show the additional contribution coming from the zero $B_{2}$ breathers in the regime $0<\xi<1 / 2$ (see Ref. [8]) does not change the universal scaling of the quantities of interest.

[83] In the Coulomb gas picture these integers correspond to magnetic and electric charges.

[84] A counter-example where $\chi_{f}(0)$ vanishes is the Richardson model (private communications with B. Gut, D. Baeriswyl, and R. Barankov). However, this model is effectively classical with suppressed quantum fluctuations. The full BCS model has positive $\chi_{f}$. 